\title{
Local Regularity Results to Nonlinear Elliptic Dirichlet Problems With Lower Order Terms
}

\author{
Francesco Clemente
}

\begin{abstract}
In this paper we study local regularity properties of weak solutions to a class of nonlinear noncoercive elliptic Dirichlet problems with $L^{1}$ datum. The model example is

$$
\begin{cases}-\Delta_{p}(w)+b(x)|D w|^{p-1}=f(x) & \text { in } \Omega, \\ w=0 & \text { on } \partial \Omega .\end{cases}
$$

Here $\Omega \subset \mathbb{R}^{N}$ is a bounded open subset, $N>1,-\Delta_{p}$ is the well known $p$-Laplace operator, $1<p<N, b$ is a function in the Lorentz space $L^{N, 1}(\Omega)$ and $f$ is a function in $L^{1}(\Omega)$. We also investigate similar issues for a lower order perturbation of these problems.
\end{abstract}

Mathematics Subject Classification (2010). 35J60; 35J25.

Keywords. Nonlinear elliptic problems, noncoercive problems, local regularity, lower order perturbation, weak solutions, $L^{1}$ datum.

\section{Introduction and main results}

Let $\Omega \subset \mathbb{R}^{N}$ be a bounded open subset, $N>1$. Let us consider the nonlinear elliptic differential operator

$$
v \mapsto-\operatorname{div}(a(x, v, D v))+B(x, D v)
$$

where $a: \Omega \times \mathbb{R} \times \mathbb{R}^{N} \rightarrow \mathbb{R}^{N}$ and $B: \Omega \times \mathbb{R}^{N} \rightarrow \mathbb{R}$ are Carathéodory mappings such that

$$
\left\{\begin{array}{l}
\exists 0<\alpha \leq \beta, 1<p<N: \\
a(x, \sigma, \xi) \cdot \xi \geq \alpha|\xi|^{p} \\
|a(x, \sigma, \xi)| \leq \beta|\xi|^{p-1}, \\
{[a(x, \sigma, \xi)-a(x, \sigma, \hat{\xi})] \cdot(\xi-\hat{\xi})>0,} \\
\text { for a.e. } x \in \Omega, \forall \sigma \in \mathbb{R}, \forall \xi \in \mathbb{R}^{N}, \xi \neq \hat{\xi}
\end{array}\right.
$$


and

$$
\left\{\begin{array}{l}
\exists b: \Omega \rightarrow \mathbb{R}: \\
|B(x, \xi)| \leq|b(x)||\xi|^{p-1}, \\
\text { for a.e. } x \in \Omega, \forall \xi \in \mathbb{R}^{N} .
\end{array}\right.
$$

The model examples of functions $a$ and $B$ we have in mind are $a(x, \sigma, \xi)=$ $|\xi|^{p-2} \xi$ and $B(x, \xi)=b(x)|\xi|^{p-1}$, respectively. The corresponding operator is a first order perturbation of the well known $p$-Laplace operator $-\Delta_{p}(v)=$ $-\operatorname{div}\left(|D v|^{p-2} D v\right)$.

If $b$ belongs to $L^{N}(\Omega)$, the mapping (1.1) defines a pseudomonotone operator acting from $W_{0}^{1, p}(\Omega)$ to its dual space ${ }^{1} W^{-1, p^{\prime}}(\Omega)$, which, in general, fails to be coercive. This feature produces specific difficulties in the study of the Dirichlet problem

$$
\begin{cases}-\operatorname{div}(a(x, w, D w))+B(x, D w)=f(x) & \text { in } \Omega \\ w=0 & \text { on } \partial \Omega,\end{cases}
$$

if no additional assumptions (as smallness conditions on the size of $\|b\|_{L^{N}(\Omega)}$ ) are required, even if the right-hand side $f$ is a smooth function on $\Omega$, since the standard theory of pseudomonotone and coercive operators (see [15]) cannot be applied.

The question of existence of solutions to (1.4) is addressed in [9] in the linear framework, that is, $p=2, a(x, \sigma, \xi)=M(x) \xi$ and $B(x, \xi)=E(x) \cdot \xi$, where $M$ is a uniformly elliptic matrix on $\Omega$ with $L^{\infty}(\Omega)$ coefficients and $E$ is a vector field on $\Omega$ such that $|E| \in L^{N}(\Omega)$. In detail, the authors prove the existence of a (unique) finite energy weak solution (i.e., which belongs to $H_{0}^{1}(\Omega)$ ) when $f \in H^{-1}(\Omega)$. This result is extended to the nonlinear case and for every value of $p \in(1, N)$ in [13].

Regularity results for weak solutions to (1.4) depending on the regularity of the datum are established in [2], [12] and [14] by means of symmetrization techniques. In particular, if $b \in L^{N}(\Omega)$, these results (see also [10]) guarantee the existence of a weak solution $w$ to (1.4) that satisfies the same regularity properties achieved in [21], [6] and [7] in the case $B \equiv 0$, that is ${ }^{2}$

$$
\begin{cases}w \in W_{0}^{1, p}(\Omega) & \text { if } m \geq\left(p^{*}\right)^{\prime}, \\ w \in W_{0}^{1,(p-1) m^{*}}(\Omega) & \text { if } \max \left\{1, \frac{N}{N(p-1)+1}\right\}<m<\left(p^{*}\right)^{\prime},\end{cases}
$$

and

$$
\begin{cases}w \in L^{\infty}(\Omega) & \text { if } m>\frac{N}{p}, \\ w \in L^{\left[(p-1) m^{*}\right]^{*}}(\Omega) & \text { if } \max \left\{1, \frac{N}{N(p-1)+1}\right\}<m<\frac{N}{p} .\end{cases}
$$

If $f$ only belongs to $L^{1}(\Omega)$ (or, more generally, $f$ is a Radon measure on $\Omega$ with bounded total variation), the problem is studied in [4] (see also [3] and $[5])$. Assuming that $b$ belongs to the Lorentz space $L^{N, 1}(\Omega)$, the existence of a renormalized solution $w$ to (1.4) (see also [11], [16] and [17]) is established

\footnotetext{
${ }^{1}$ For every $1<q<\infty, q^{\prime}$ denotes the Hölder conjugate of $q$, that is, $q^{\prime}=\frac{q}{q-1}$.

${ }^{2}$ For every $1 \leq q<N, q^{*}$ denotes the Sobolev conjugate of $q$, that is, $q^{*}=\frac{N q}{N-q}$.
} 
performing an approximation procedure. This solution satisfies the equation in (1.4) in the distributional sense and, if $p>2-\frac{1}{N}$,

$$
w \in W_{0}^{1, q}(\Omega) \quad \forall 1 \leq q<N^{\prime}(p-1) .
$$

The first aim of this paper is to study the behaviour of the solution obtained in [4] "far" from the singularities of the datum, in the spirit of [8]. More precisely, we assume that

$$
\begin{aligned}
& p>2-\frac{1}{N}, \\
& f \in L^{1}(\Omega),
\end{aligned}
$$

and

$$
\exists U \subset \subset \Omega, m>1: \quad f \in L^{m}(\Omega \backslash U) .
$$

As happens in the case $B \equiv 0$, we expect that, even if $w$ only satisfies (1.7), there is an improvement in the regularity properties of $w$ and its distributional gradient $D w$ depending on the regularity properties of $f$ away from $U$. The result is the following.

Theorem 1.1. Assume (1.2), (1.3) with $b \in L^{N, 1}(\Omega)$ and (1.8)-(1.10). Let $V \subset \subset \Omega$ be such that $V \supset \bar{U}$. Then, there exists a weak solution $w$ to (1.4) such that

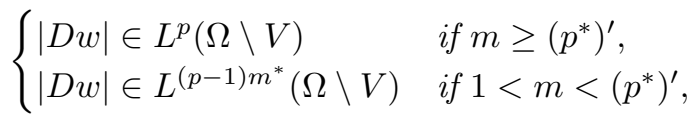

and

$$
\begin{cases}w \in L^{\infty}(\Omega \backslash V) & \text { if } m>\frac{N}{p} \\ w \in L^{\left[(p-1) m^{*}\right]^{*}}(\Omega \backslash V) & \text { if } 1<m<\frac{N}{p} .\end{cases}
$$

We emphasize that our result concern solutions obtained as limit of approximations and that satisfy the equation in (1.4) in the distributional sense. The enhanced regularity is not true for every distributional solution to (1.4) when the datum is only a function in $L^{1}(\Omega)$. As a matter of fact, a classical counterexample in [18] shows that there is no uniqueness of the distributional solution to (1.4) outside $W_{0}^{1, p}(\Omega)$. Moreover, the regularity properties in the statement of Theorem 1.1 are false for the "pathological" solution of the quoted counterexample.

Then, we consider the following lower perturbation of (1.4):

$$
\begin{cases}-\operatorname{div}(a(x, u, D u))+B(x, D u)+K(x, u)=f(x) & \text { in } \Omega, \\ u=0 & \text { on } \partial \Omega,\end{cases}
$$

where $K: \Omega \times \mathbb{R} \rightarrow \mathbb{R}$ is a Carathéodory function such that

$$
\sup _{|\tau| \leq \sigma}|K(\cdot, \tau)| \in L^{1}(\Omega) \quad \forall \sigma>0
$$


and

$$
\left\{\begin{array}{l}
\exists k \in L^{1}(\Omega) \text { positive on } \Omega, \lambda>1: \\
K(x, \sigma) \operatorname{sign}(\sigma) \geq k(x)|\sigma|^{\lambda}, \\
\text { for a.e. } x \in \Omega, \forall t \in \mathbb{R} .
\end{array}\right.
$$

The model example of function $K$ we have in mind is $K(x, \sigma)=k(x)|\sigma|^{\lambda-1} \sigma$.

The existence of a weak solution $u$ that satisfies (1.7), when $b \in L^{N, 1}(\Omega)$, and (1.5)-(1.6), when $b \in L^{N}(\Omega)$, is guaranteed also for the problem (1.11), because of the coercivity properties of the zero order term $K(x, u)$. Moreover, if $k$ satisfies

$$
\exists h>0: \quad k^{-h} \in L^{1}(\Omega),
$$

then a twofold regularizing effect on $u$ occurs (see [10]): on the one hand, $u$ satisfies better regularity properties than (1.5)-(1.7); on the other hand, the regularity properties (1.5)-(1.7) still hold for $u$ even if $b \notin L^{N}(\Omega)$. In detail, assuming that $b \in L^{r}(\Omega)$ for some $p<r<N$ and $f \in L^{m}(\Omega)$ for some $m \geq 1$, the author prove that

$$
\begin{cases}u \in W_{0}^{1, p}(\Omega) & \text { if } m>1, \lambda \geq \bar{\lambda} \\ u \in W_{0}^{1, \tilde{q}}(\Omega) & \text { if } m>1, \underline{\lambda}<\lambda<\bar{\lambda}, \\ u \in W_{0}^{1, q}(\Omega) \quad \forall 1 \leq q<\tilde{q}_{1} & \text { if } m=1, \lambda>\underline{\lambda}\end{cases}
$$

and

where

$$
\begin{cases}K(\cdot, u)|u|^{\tilde{\lambda}-\lambda} \in L^{1}(\Omega) & \text { if } m>1, \lambda>\underline{\lambda} \\ K(\cdot, u) \in L^{1}(\Omega) & \text { if } m=1, \lambda>\underline{\lambda}\end{cases}
$$

$$
\begin{gathered}
\underline{\lambda}=\frac{(p-1)(h+1) r}{(r-p) h}, \\
\bar{\lambda}=\max \left\{\frac{[(p-1) r+p] h+p r}{(r-p) h}, \frac{h+m}{(m-1) h}\right\}, \\
\tilde{\lambda}=\min \left\{\frac{(\lambda-p+1)(h+1) r}{p h+r}, \frac{\lambda(h+1) m}{h+m}\right\}, \\
\tilde{q}=\min \left\{\frac{(\lambda-p+1) h r}{(\lambda+1) h+r}, \frac{p \lambda h m}{(\lambda+1) h+m}\right\}, \\
\tilde{q}_{1}=\frac{p \lambda h}{(\lambda+1) h+1} .
\end{gathered}
$$

Thus, it seems natural to investigate what happens locally. In this connection, here we proceed in two slightly different directions. The first one consists in assuming (1.14) and studying a "local" version of regularity results (1.15)-(1.16). The result is the following.

Theorem 1.2. Assume (1.2), (1.3) with $b \in L^{r}(\Omega)$ for some $p<r<N$, (1.9), (1.10) and (1.12)-(1.14). Assume also that $\lambda>\underline{\lambda}$ and let $V \subset \subset \Omega$ be such 
that $V \supset \bar{U}$. Then, there exists a weak solution $u$ to (1.11) such that

$$
\begin{cases}|D u| \in L^{p}(\Omega \backslash V) & \text { if } \lambda \geq \bar{\lambda}, \\ |D u| \in L^{\tilde{q}}(\Omega \backslash V) & \text { if } \underline{\lambda}<\lambda<\bar{\lambda},\end{cases}
$$

and

$$
K(\cdot, u)|u|^{\tilde{\lambda}-\lambda} \in L^{1}(\Omega \backslash V)
$$

We also investigate the regularizing effect of the term $K(x, u)$ on $u$ replacing assumption (1.14) with its own "localized" counterpart:

$$
\exists U \subset \subset \Omega, h>0: \quad k^{-h} \in L^{1}(\Omega \backslash U) .
$$

We remark that, in this case, we have to assume (1.8) and that $b \in L^{N, 1}(\Omega)$, which is a stronger condition than $b \in L^{r}(\Omega)$ with $p<r<N$. Therefore, the quantities $\underline{\lambda}, \bar{\lambda}, \tilde{\lambda}$ and $\tilde{q}$ which appear in the following statement (as in the statement of Theorem 3.2 and Lemma 3.4 below), are as in (1.17)-(1.20) but with $r=N$.

Theorem 1.3. Assume (1.2), (1.3) with $b \in L^{N, 1}(\Omega)$, (1.8), (1.9), (1.12), (1.13) and (1.22). Assume also that $\lambda>\underline{\lambda}$, where $\underline{\lambda}$ is as in (1.17) but with $r=N$. Let $V \subset \subset \Omega$ be such that $V \supset \bar{U}$. Then, there exists a weak solution $u$ to (1.4) such that

$$
|D u| \in L^{\tilde{q}_{1}}(\Omega \backslash V), \quad K(\cdot, u) \in L^{1}(\Omega \backslash V) .
$$

Moreover, if $f$ satisfies (1.10), then

$$
\begin{cases}|D u| \in L^{p}(\Omega \backslash V) & \text { if } \lambda \geq \bar{\lambda}, \\ |D u| \in L^{\tilde{q}}(\Omega \backslash V) & \text { if } \underline{\lambda}<\lambda<\bar{\lambda},\end{cases}
$$

and

$$
K(\cdot, u)|u|^{\tilde{\lambda}-\lambda} \in L^{1}(\Omega \backslash V),
$$

where $\bar{\lambda}, \tilde{\lambda}$ and $\tilde{q}$ are as in (1.18)-(1.20) but with $r=N$.

Let us finally describe the plan of the paper. In Section 2 we first recall the construction of the weak solution $w$ to (1.4) obtained in [4] by means of approximations. If $\left\{w_{n}\right\}$ is a sequence of regular solutions to suitable approximate problems (see (2.3) below) that converges (in some sense) to $w$, in order to prove Theorem 1.1 the main point is to get suitable local estimates on $w_{n}$ and $D w_{n}$. Then, the result is deduced immediately putting together these estimates with the convergence properties of $w_{n}$. The same outline is followed in Section 3 to prove Theorems 1.2 and 1.3.

\section{Local regularity results for (1.4)}

Let $f \in L^{1}(\Omega)$. We recall that a function $w: \Omega \rightarrow \mathbb{R}$ is a weak solution of (1.4) if $w$ satisfies

$$
w \in W_{0}^{1,1}(\Omega), \quad|a(\cdot, w, D w)| \in L_{\mathrm{loc}}^{1}(\Omega), \quad B(\cdot, D w) \in L_{\mathrm{loc}}^{1}(\Omega),
$$


and

$$
\int_{\Omega} a(x, w, D w) \cdot D \varphi+\int_{\Omega} B(x, D w) \varphi=\int_{\Omega} f(x) \varphi \quad \forall \varphi \in C_{c}^{\infty}(\Omega) .
$$

The existence of a weak solution $w$ to (1.4) is established in [4] assuming that $b$ belongs to the Lorentz space $L^{N, 1}(\Omega)$, namely $b$ satisfies

$$
\int_{0}^{|\Omega|} b^{*}(\sigma) \sigma^{\frac{1}{N}} \frac{d \sigma}{\sigma}<\infty
$$

where $b^{*}$ is the decreasing rearrangement of $b$, that is, the decreasing function defined by

$$
b^{*}(\sigma)=\inf \{\tau \geq 0:|\{x \in \Omega:|b(x)|>\tau\}|<\sigma\} \quad \forall \sigma \in[0,|\Omega|] .
$$

Let us recall the construction of $w$. Consider the following family of approximate problems $(n \in \mathbb{N})$ :

$$
\begin{cases}-\operatorname{div}\left(a\left(x, w_{n}, D w_{n}\right)\right)+B_{n}\left(x, D w_{n}\right)=f_{n}(x) & \text { in } \Omega \\ w_{n}=0 & \text { on } \partial \Omega\end{cases}
$$

where $B_{n}$ and $f_{n}$ are the truncations at level $n$ of $B$ and $f$, respectively. More precisely, we define

$$
\left\{\begin{array}{l}
B_{n}(x, \xi)=T_{n}(B(x, \xi)), \\
f_{n}(x)=T_{n}(f(x)) \\
\text { for a.e. } x \in \Omega
\end{array}\right.
$$

where, for any $\sigma>0, T_{\sigma}$ denotes the function defined by

$$
T_{\sigma}(s)=\max \{-\sigma, \min \{s, \sigma\}\} \quad \forall s \in \mathbb{R} .
$$

Clearly, we have that

$$
\left\{\begin{array}{l}
\left|B_{n}(x, \xi)\right| \leq \min \{|B(x, \xi)|, n\} \\
\left|f_{n}(x)\right| \leq \min \{|f(x)|, n\} \\
\text { for a.e. } x \in \Omega, \forall \xi \in \mathbb{R}^{N}, \forall n \in \mathbb{N} .
\end{array}\right.
$$

It is well known (see [15] and [21]) that for every $n \in \mathbb{N}$ there exists a weak solution $w_{n} \in W_{0}^{1, p}(\Omega) \cap L^{\infty}(\Omega)$ to (1.4) and that $w_{n}$ satisfies

$$
\int_{\Omega} a\left(x, w_{n}, D w_{n}\right) \cdot D v+\int_{\Omega} B_{n}\left(x, D w_{n}\right) v=\int_{\Omega} f_{n}(x) v \quad \forall v \in W_{0}^{1, p}(\Omega) .
$$

Then, one has that

$$
\begin{cases}\left\{w_{n}\right\} & \text { is bounded in } M^{\frac{p^{*}}{p^{\prime}}}(\Omega), \\ \left\{\left|D w_{n}\right|\right\} & \text { is bounded in } M^{N^{\prime}}(p-1)(\Omega),\end{cases}
$$

where, for any $q>0, M^{q}(\Omega)$ denotes the Marcinkiewicz space of all measurable functions $v: \Omega \rightarrow \mathbb{R}$ such that

$$
\exists C>0:|\{x \in \Omega:|v(x)|>\sigma\}| \leq \frac{C}{\sigma^{q}} \quad \forall \sigma>0 .
$$


In particular, if $p>2-\frac{1}{N}$, it follows that

$$
\left\{w_{n}\right\} \text { is bounded in } W_{0}^{1, q}(\Omega) \quad \forall 1 \leq q<N^{\prime}(p-1) .
$$

Hence, there exists a function $w$ that satisfies (1.7) and such that, up to a subsequence, $w_{n} \rightarrow w$ a.e. in $\Omega$. Moreover, one has that $D w_{n} \rightarrow D w$ a.e. in $\Omega$. Thanks to assumptions (1.2) and (1.3), it follows that $a\left(\cdot, w_{n}, D w_{n}\right) \rightarrow$ $a(\cdot, w, D w)$ and $B_{n}\left(\cdot, D w_{n}\right) \rightarrow B(\cdot, D w)$ in $\left(L^{1}(\Omega)\right)^{N}$ and $L^{1}(\Omega)$, respectively. Therefore, passing to the limit in (2.3), it results that $w$ is a weak solution to $(1.4)$.

Now, assume that $f \in L^{m}(\Omega \backslash U)$ for some $U \subset \subset \Omega$ and $m>1$. By means of standard regularization techniques, it is possible to construct a function $\psi \in W^{1, \infty}(\Omega)$ with $0 \leq \psi \leq 1$ in $\Omega$, such that

$$
\psi= \begin{cases}0 & \text { in } \bar{U}, \\ 1 & \text { in } \Omega \backslash V,\end{cases}
$$

where $V \subset \subset \Omega$ is such that $V \supset \bar{U}$. In particular, assumption (1.10) implies the following condition:

$$
\left\{\begin{array}{l}
\exists \psi \in W^{1, \infty}(\Omega), m>1: \\
0 \leq \psi \leq 1 \quad \text { in } \Omega \\
f \psi \in L^{m}(\Omega)
\end{array}\right.
$$

Hence, Theorem 1.1 is an immediate consequence of the following result.

Theorem 2.1. Assume (1.2), (1.3) with $b \in L^{N, 1}(\Omega)$ and (1.8), (1.9) and (2.10). Then, there exist a weak solution $w$ to (1.4) and $\eta_{0}>1$ which depends only on $\psi, m, N$ and $p$, such that

$$
\left\{\begin{array}{lll}
w \psi^{\eta_{0}} \in W_{0}^{1, p}(\Omega) \cap L^{\infty}(\Omega) & & \text { if } m>\frac{N}{p}, \\
w \psi^{\eta_{0}} \in W_{0}^{1, p}(\Omega) \cap L^{\left[(p-1) m^{*}\right]^{*}}(\Omega) & & \text { if }\left(p^{*}\right)^{\prime} \leq m<\frac{N}{p}, \\
w \psi^{\eta_{0}} \in W_{0}^{1,(p-1) m^{*}}(\Omega) & & \text { if } 1<m<\left(p^{*}\right)^{\prime} .
\end{array}\right.
$$

Now, we observe that in order to prove Theorem 2.1 it is sufficient to get suitable estimates on the sequences $\left\{w_{n} \psi^{\eta}\right\}$ and $\left\{\left|D\left(w_{n} \psi^{\eta}\right)\right|\right\}$ for some $\eta>1$, since $w_{n} \rightarrow w$ and $D w_{n} \rightarrow D w$ a.e. in $\Omega$.

\subsection{Local estimates on $w_{n}$}

Our starting point is the fact that, by Sobolev inequality ${ }^{3},(2.8)$ implies that $^{2}$

$$
\left\{w_{n}\right\} \text { is bounded in } L^{s}(\Omega) \quad \forall 1 \leq s<\frac{p^{*}}{p^{\prime}} .
$$

This (global) estimate plays a crucial role in proving the following.

\footnotetext{
${ }^{3}$ We recall that, by Sobolev inequality, there exists a positive constant $\mathcal{S}_{0}$ which depends only on $N$ and $p$, such that

$$
\|v\|_{L^{p^{*}}(\Omega)} \leq \mathcal{S}_{0}\||D v|\|_{L^{p}(\Omega)} \quad \forall v \in W_{0}^{1, p}(\Omega) .
$$
}


Lemma 2.2. Assume (1.2), (1.3) with $b \in L^{N, 1}(\Omega)$, (1.8), (1.9) and (2.10) with $1<m<\frac{N}{p}$. Then, there exists $\eta_{1}>\frac{1}{p-1}$ which depends only on $\psi, m$, $N$ and $p$, such that the sequence $\left\{w_{n} \psi^{\eta_{1}}\right\}$ is bounded in $L^{\left[(p-1) m^{*}\right]^{*}}(\Omega)$.

Proof. We follow the approach of [6] (see also [8]) and we divide the proof into four steps.

STEP I. First, let $\phi \in W^{1, \infty}(\Omega)$ be such that $0 \leq \phi \leq \psi$ in $\Omega$. We observe that $f \phi \in L^{m}(\Omega)$, by (2.10). Then, we fix $\frac{1}{p^{\prime}}<\gamma<\frac{\left[(p-1) m^{*}\right]^{*}}{p^{*}}, \delta>0$, $\eta=p+p^{\prime} \gamma$ and we choose

$$
v_{\delta} \phi^{\eta}=\left[\left(\delta+\left|w_{n}\right|\right)^{p(\gamma-1)+1}-\delta^{p(\gamma-1)+1}\right] \operatorname{sign}\left(w_{n}\right) \phi^{\eta}
$$

as a test function in (2.7). Notice that $m>1$ and $\gamma>\frac{1}{p^{\prime}}$ implies that $\frac{1}{p^{\prime}}<\frac{\left[(p-1) m^{*}\right]^{*}}{p^{*}}$ and $p(\gamma-1)+1>0$, respectively. Since

$$
D\left(v_{\delta} \phi^{\eta}\right)=[p(\gamma-1)+1] D w_{n}\left(\delta+\left|w_{n}\right|\right)^{p(\gamma-1)} \phi^{\eta}+\eta D \phi v_{\delta} \phi^{\eta-1},
$$

exploiting (1.2), (2.6) and (1.3), we obtain that

$$
\begin{aligned}
\alpha[p(\gamma-1)+1] \int_{\Omega}\left|D w_{n}\right|^{p}\left(\delta+\left|w_{n}\right|\right)^{p(\gamma-1)} \phi^{\eta} & \\
\leq \beta \eta\||D \phi|\|_{L^{\infty}(\Omega)} \int_{\Omega}\left|D w_{n}\right|^{p-1}\left|v_{\delta}\right| \phi^{\eta-1}+\int_{\Omega}|b(x)|\left|D w_{n}\right|^{p-1}\left|v_{\delta}\right| \phi^{\eta} & +\int_{\Omega}|f(x)|\left|v_{\delta}\right| \phi^{\eta}
\end{aligned}
$$

Thanks to Young inequality, the first term on the right-hand side of (2.12) can be estimated by

$$
\begin{aligned}
\frac{\alpha[p(\gamma-1)+1]}{p^{\prime}} \int_{\Omega}\left|D w_{n}\right|^{p}(\delta & \left.+\left|w_{n}\right|\right)^{p(\gamma-1)} \phi^{\eta} \\
& +\frac{\left(\beta \eta|||D \phi| \|_{L^{\infty}(\Omega)}\right)^{p}}{p \alpha^{p-1}[p(\gamma-1)+1]^{p-1}} \int_{\Omega}\left(\delta+\left|w_{n}\right|\right)^{p \gamma} \phi^{\eta-p} .
\end{aligned}
$$

Hence, it follows that

$$
\begin{aligned}
& C_{0} \int_{\Omega}\left|D w_{n}\right|^{p}\left(\delta+\left|w_{n}\right|\right)^{p(\gamma-1)} \phi^{\eta} \\
& \leq C_{1} \int_{\Omega}\left(\delta+\left|w_{n}\right|\right)^{p \gamma} \phi^{\eta-p}+\int_{\Omega}|b(x)|\left|D w_{n}\right|^{p-1}\left|v_{\delta}\right| \phi^{\eta} \\
&+\int_{\Omega}|f(x)|\left|v_{\delta}\right| \phi^{\eta}
\end{aligned}
$$

where $C_{0}=\frac{\alpha[p(\gamma-1)+1]}{p}$ and $C_{1}=\frac{\left(\beta \eta\||D \phi|\|_{L^{\infty}(\Omega)}\right)^{p}}{p\{\alpha[p(\gamma-1)+1]\}^{p-1}}$.

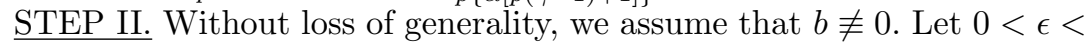
$\|b\|_{L^{N}(\Omega)}$ and let $U_{0} \supset \Omega$ be a cube. We extend $b$ and $w_{n}$ to vanish outside $\Omega$. By bisection of the edges of $U_{0}$, we subdivide $U_{0}$ into $2^{N}$ congruent subcubes with disjoint interiors. If there is a subcube $U$ such that $\|b\|_{L^{N}(U)}>\epsilon$, then all 
subcubes are similarly subdivided. The process terminates in a finite number of steps, otherwise there would be an infinite sequence of nested subcubes $U_{j+1} \subset U_{j} \subset U_{0}$ such that $\left|U_{j}\right|=\frac{\left|U_{0}\right|}{2^{j N}}$ and $\|b\|_{L^{N}\left(U_{j}\right)}>\epsilon$ for every $j \in \mathbb{N}$, which is a contradiction, since $\|b\|_{L^{N}\left(U_{j}\right)} \rightarrow 0$ as $j \rightarrow \infty$, by the absolute continuity of the integral. Thus, there exists a finite number of congruent subcubes $U_{1}, \ldots, U_{l_{\epsilon}} \subset U_{0}$ with disjoint interiors and such that

$$
\begin{gathered}
\Omega \subset U_{0}=U_{1} \cup \ldots \cup U_{l_{\epsilon}}, \\
\|b\|_{L^{N}\left(U_{1}\right)}, \ldots,\|b\|_{L^{N}\left(U_{l_{\epsilon}}\right)} \leq \epsilon .
\end{gathered}
$$

Then, using (2.14), (2.15) and Hölder inequality, we have that

$$
\begin{aligned}
& \int_{\Omega}|b(x)|\left|D w_{n}\right|^{p-1}\left|v_{\delta}\right| \phi^{\eta} \\
& \leq \epsilon \sum_{j=1}^{l_{\epsilon}}\left[\int_{U_{j}}\left|D w_{n}\right|^{p}\left(\delta+\left|w_{n}\right|\right)^{p(\gamma-1)} \phi^{\eta}\right]^{\frac{1}{p^{\prime}}}\left[\int_{U_{j}}\left(\delta+\left|w_{n}\right|\right)^{p^{*} \gamma} \phi^{\frac{p^{*} \eta}{p}}\right]^{\frac{1}{p^{*}}} .
\end{aligned}
$$

Furthermore, thanks to Sobolev inequality ${ }^{4}$ and the fact that $\left|U_{j}\right|=\frac{\left|U_{0}\right|}{l_{\epsilon}}$, for every $j=1, \ldots, l_{\epsilon}$ we have that

$$
\begin{aligned}
& {\left[\int_{U_{j}}\left(\delta+\left|w_{n}\right|\right)^{p^{*} \gamma} \phi^{\frac{p^{*} \eta}{p}}\right]^{\frac{1}{p^{*}}} } \\
\leq & \mathcal{S}\left(\frac{l_{\epsilon}}{\left|K_{0}\right|}\right)^{\frac{1}{N}}\left[\int_{U_{j}}\left(\delta+\left|w_{n}\right|\right)^{p \gamma} \phi^{\eta}\right]^{\frac{1}{p}}+\mathcal{S}\left\{\int_{U_{j}}\left|D\left[\left(\delta+\left|w_{n}\right|\right)^{\gamma} \phi^{\frac{\eta}{p}}\right]\right|^{p}\right\}^{\frac{1}{p}} .
\end{aligned}
$$

Hence, the right-hand side of (2.16) can be estimated by

$$
\begin{array}{r} 
\\
\mathcal{S}\left(\frac{l_{\epsilon}}{|\Omega|}\right)^{\frac{1}{N}} \epsilon \sum_{j=1}^{l_{\epsilon}}\left[\int_{U_{j}}\left|D w_{n}\right|^{p}\left(\delta+\left|w_{n}\right|\right)^{p(\gamma-1)} \phi^{\eta}\right]^{\frac{1}{p^{\prime}}}\left[\int_{U_{j}}\left(\delta+\left|w_{n}\right|\right)^{p \gamma} \phi^{\eta}\right]^{\frac{1}{p}} \\
+\mathcal{S} \epsilon \sum_{j=1}^{l_{\epsilon}}\left[\int_{U_{j}}\left|D w_{n}\right|^{p}\left(\delta+\left|w_{n}\right|\right)^{p(\gamma-1)} \phi^{\eta}\right]^{\frac{1}{p^{\prime}}}\left\{\int_{U_{j}}\left|D\left[\left(\delta+\left|w_{n}\right|\right)^{\gamma} \phi^{\frac{\eta}{p}}\right]\right|^{p}\right\}^{\frac{1}{p}},
\end{array}
$$

${ }^{4}$ We recall that, by Sobolev inequality, there exists a positive constant $\mathcal{S}$ which depends only on $N$ and $p$, such that (see [1])

$$
\|v\|_{L^{p^{*}}(U)} \leq \mathcal{S}\left[\frac{1}{|U|^{\frac{1}{N}}}\|v\|_{L^{p}(U)}+\|\mid D v\|_{L^{p}(U)}\right] \quad \forall \text { cube } U \subset \mathbb{R}^{N}, \forall v \in W^{1, p}(U) .
$$


which in turn, by Hölder inequality and (2.14) again, is controlled by

$$
\begin{aligned}
& \mathcal{S}\left(\frac{l_{\epsilon}}{|\Omega|}\right)^{\frac{1}{N}} \epsilon\left[\int_{\Omega}\left|D w_{n}\right|^{p}\left(\delta+\left|w_{n}\right|\right)^{p(\gamma-1)} \phi^{\eta}\right]^{\frac{1}{p^{\prime}}}\left[\int_{\Omega}\left(\delta+\left|w_{n}\right|\right)^{p \gamma} \phi^{\eta}\right]^{\frac{1}{p}} \\
& \quad+\mathcal{S} \epsilon\left[\int_{\Omega}\left|D w_{n}\right|^{p}\left(\delta+\left|w_{n}\right|\right)^{p(\gamma-1)} \phi^{\eta}\right]^{\frac{1}{p^{\prime}}}\left\{\int_{\Omega}\left|D\left[\left(\delta+\left|w_{n}\right|\right)^{\gamma} \phi^{\frac{\eta}{p}}\right]\right|^{p}\right\}^{\frac{1}{p}} .
\end{aligned}
$$

Since

$$
\begin{aligned}
\left|D\left[\left(\delta+\left|w_{n}\right|\right)^{\gamma} \phi^{\frac{\eta}{p}}\right]\right| \leq \gamma\left|D w_{n}\right|(\delta & \left.+\left|w_{n}\right|\right)^{\gamma-1} \phi^{\frac{\eta}{p}} \\
& +\frac{\eta\||D \phi|\|_{L^{\infty}(\Omega)}}{p}\left(\delta+\left|w_{n}\right|\right)^{\gamma} \phi^{\frac{\eta-p}{p}}
\end{aligned}
$$

using Young inequality and the fact that $0 \leq \phi \leq 1$ in $\Omega$, putting together (2.16) and (2.17) we obtain that

$$
\begin{aligned}
& \int_{\Omega}|b(x)|\left|D w_{n}\right|^{p-1}\left|v_{\delta}\right| \phi^{\eta} \\
& \quad \leq C_{2} \epsilon \int_{\Omega}\left|D w_{n}\right|^{p}\left(\delta+\left|w_{n}\right|\right)^{p(\gamma-1)} \phi^{\eta}+C_{3} \epsilon \int_{\Omega}\left(\delta+\left|w_{n}\right|\right)^{p \gamma} \phi^{\eta-p}
\end{aligned}
$$

where $C_{2}=\mathcal{S}\left(\frac{1}{p^{\prime}|\Omega|^{\frac{1}{N}}}+\frac{\eta\||D \phi|\|_{L^{\infty}(\Omega)}}{p p^{\prime}}+\gamma\right), C_{3}=\mathcal{S}\left(\frac{l_{\epsilon}^{\frac{p}{N}}}{p|\Omega|^{\frac{1}{N}}}+\frac{\eta\||D \phi|\|_{L} \infty(\Omega)}{p^{2}}\right)$.

Now, we choose $\epsilon$ such that $C_{2} \epsilon=\frac{C_{0}}{2}$, that is, $\epsilon=\frac{C_{0}}{2 C_{2}}$. In this way, from (2.13) and (2.19) we deduce that

$$
\begin{aligned}
\int_{\Omega}\left|D w_{n}\right|^{p}\left(\delta+\left|w_{n}\right|\right)^{p(\gamma-1)} \phi^{\eta} & \\
& \leq C_{4} \int_{\Omega}\left(\delta+\left|w_{n}\right|\right)^{p \gamma} \phi^{\eta-p}+C_{5} \int_{\Omega}|f(x)|\left|v_{\delta}\right| \phi^{\eta}
\end{aligned}
$$

where $C_{4}=\frac{C_{3}}{C_{2}}+\frac{2 C_{1}}{C_{0}}$ and $C_{5}=\frac{2}{C_{0}}$. Then, in virtue of Sobolev inequality and (2.18), estimate (2.20) yields

$$
\begin{array}{r}
\left\{\int_{\Omega}\left[\left(\delta+\left|w_{n}\right|\right)^{\gamma}-\delta^{\gamma}\right]^{p^{*}} \phi^{\frac{p^{*} \eta}{p}}\right\}^{\frac{p}{p^{*}}} \leq \mathcal{S}_{0}^{p} \int_{\Omega}\left|D\left\{\left[\left(\delta+\left|w_{n}\right|\right)^{\gamma}-\delta^{\gamma}\right] \phi^{\frac{\eta}{p}}\right\}\right|^{p} \\
\leq C_{6} \int_{\Omega}\left(\delta+\left|w_{n}\right|\right)^{p \gamma} \phi^{\eta-p}+C_{7} \int_{\Omega}|f(x)|\left|v_{\delta}\right| \phi^{\eta}
\end{array}
$$

which in turn, letting $\delta \rightarrow 0$ and applying dominate convergence Theorem, implies that

$$
\begin{aligned}
\left(\int_{\Omega}\left|w_{n}\right|^{p^{*} \gamma} \phi^{\frac{p^{*} \eta}{p}}\right. & )^{\frac{p}{p^{*}}} \\
& \leq C_{6} \int_{\Omega}\left|w_{n}\right|^{p \gamma} \phi^{\eta-p}+C_{7} \int_{\Omega}|f(x)|\left|w_{n}\right|^{p(\gamma-1)+1} \phi^{\eta}
\end{aligned}
$$


where $C_{6}=\left(2 \mathcal{S}_{0}\right)^{p} C_{4}+\left(\frac{2 \mathcal{S}_{0} \eta\||D \phi|\|_{L} \infty(\Omega)}{p}\right)^{p}$ and $C_{7}=\left(2 \mathcal{S}_{0}\right)^{p} C_{5}$.

STEP III. By Hölder inequality, we have that

$$
\int_{\Omega}\left|f(x)\left\|\left.w_{n}\right|^{p(\gamma-1)+1} \phi^{\eta} \leq\right\| f \phi \|_{L^{m}(\Omega)}\left[\int_{\Omega}\left|w_{n}\right|^{[p(\gamma-1)+1] m^{\prime}} \phi^{(\eta-1) m^{\prime}}\right]^{\frac{1}{m^{\prime}}} .\right.
$$

Since $\gamma<\frac{\left[(p-1) m^{*}\right]^{*}}{p^{*}}$ implies that $[p(\gamma-1)+1] m^{\prime}<p^{*} \gamma$, by Hölder inequality again, from (2.21) we obtain that

$$
\begin{aligned}
& \left(\int_{\Omega}\left|w_{n}\right|^{p^{*} \gamma} \phi^{\frac{p^{*} \eta}{p}}\right)^{\frac{p}{p^{*}}} \\
& \quad \leq C_{6} \int_{\Omega}\left|w_{n}\right|^{p \gamma} \phi^{\eta-p}+C_{8}\|f \phi\|_{L^{m}(\Omega)}\left(\int_{\Omega}\left|w_{n}\right|^{p^{*} \gamma} \phi^{\frac{p^{*} \gamma(\eta-1)}{p(\gamma-1)+1}}\right)^{\frac{[p(\gamma-1)+1]}{p^{*} \gamma}},
\end{aligned}
$$

where $C_{8}=|\Omega|^{\frac{1}{m^{\prime}}}-\frac{p(\gamma-1)+1}{p^{*} \gamma} C_{7}$. Furthermore, since $\eta=p+p^{\prime} \gamma>p^{\prime} \gamma$ implies that $\frac{p^{*} \eta}{p}<\frac{p^{*} \gamma(\eta-1)}{p(\gamma-1)+1}$, exploiting the fact that $0 \leq \phi \leq 1$ in $\Omega$ and using Young inequality, we have that

$$
\begin{aligned}
C_{8}\|f \phi\|_{L^{m}(\Omega)} & \left(\int_{\Omega}\left|w_{n}\right|^{p^{*} \gamma} \phi^{\frac{p^{*} \gamma(\eta-1)}{p(\gamma-1)+1}}\right)^{\frac{p(\gamma-1)+1}{p^{*} \gamma}} \\
& \leq C_{8}\|f \phi\|_{L^{m}(\Omega)}\left(\int_{\Omega}\left|w_{n}\right|^{p^{*} \gamma} \phi^{\frac{p^{*} \eta}{p}}\right)^{\frac{p(\gamma-1)+1}{p^{*} \gamma}} \\
& \leq \frac{C_{8}^{p^{\prime} \gamma}}{p^{\prime} \gamma}\|f \phi\|_{L^{p^{\prime}}(\Omega)}^{p}+\frac{p(\gamma-1)+1}{p \gamma}\left(\int_{\Omega}\left|w_{n}\right|^{p^{*} \gamma} \phi^{\frac{p^{*} \eta}{p}}\right)^{\frac{p}{p^{*}}}
\end{aligned}
$$

Thus, from (2.22) we get

$$
\left(\int_{\Omega}\left|w_{n} \phi^{\frac{\eta}{p \gamma}}\right|^{p^{*} \gamma}\right)^{\frac{p}{p^{*}}} \leq C_{9} \int_{\Omega}\left|w_{n} \phi^{\frac{\eta-p}{p \gamma}}\right|^{p \gamma}+C_{10}\|f \phi\|_{L^{m}(\Omega)}^{p^{\prime} \gamma},
$$

where $C_{9}=p^{\prime} \gamma C_{6}$ and $C_{10}=C_{8}^{p^{\prime} \gamma}$.

Recalling the choice of $\eta=p+p^{\prime} \gamma$, the previous inequality becomes

$$
\int_{\Omega}\left|w_{n} \phi^{\frac{1}{p-1}+\frac{1}{\gamma}}\right|^{p^{*} \gamma} \leq C_{11}(\gamma)\left[\left(\int_{\Omega}\left|w_{n} \phi^{\frac{1}{p-1}}\right|^{p \gamma}\right)^{\frac{p^{*}}{p}}+\|f \phi\|_{L^{m}(\Omega)}^{\frac{p^{*} \gamma}{p-1}}\right]
$$

where $C_{11}(\gamma)=\max \left\{\left(2 C_{9}(\gamma)\right)^{\frac{p^{*}}{p}},\left(2 C_{10}(\gamma)\right)^{\frac{p^{*}}{p}}\right\}$. We remark that

$$
\begin{gathered}
C_{9}(\gamma)=C_{12} \gamma\left\{1+\frac{\gamma^{p}}{[p(\gamma-1)+1]^{p}}\right\} \\
C_{10}(\gamma)=C_{13}^{p^{\prime} \gamma}|\Omega|^{\frac{p^{\prime} \gamma}{m^{\prime}}-\frac{p^{\prime}[p(\gamma-1)+1]}{p^{*}}}\left[\frac{\gamma^{p}}{p(\gamma-1)+1}\right]^{p^{\prime} \gamma},
\end{gathered}
$$


where $C_{12}$ and $C_{13}$ are positive constants that do not depend on $\gamma$. Hence, $C_{11}(\gamma)$ depends continuously on $\gamma$ and satisfies

$$
\lim _{\gamma \rightarrow \frac{1}{p^{\prime}}} C_{11}(\gamma)=\infty, \quad \lim _{\gamma \rightarrow \frac{\left[(p-1) m^{*}\right]^{*}}{p^{*}}} C_{11}(\gamma) \in(0, \infty)
$$

In particular, passing to the limit as $\gamma \rightarrow \frac{\left[(p-1) m^{*}\right]^{*}}{p^{*}}$ in $(2.23)$ and using dominate convergence Theorem, we deduce that estimate (2.23) holds for every $\frac{1}{p^{\prime}}<\gamma \leq \frac{\left[(p-1) m^{*}\right]^{*}}{p^{*}}$.

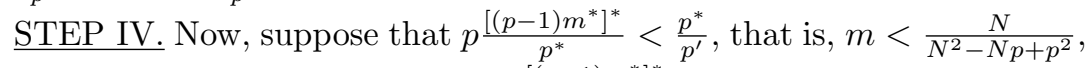
and consider estimate (2.23) with $\gamma=\frac{\left[(p-1) m^{*}\right]^{*}}{p^{*}}$ and $\phi=\psi$ :

$$
\begin{aligned}
\int_{\Omega}\left|w_{n} \psi^{\frac{1}{p-1}}+\frac{p^{*}}{\left[(p-1) m^{*}\right]^{*}}\right|^{\left[(p-1) m^{*}\right]^{*}} \\
\leq C_{11}\left[\left(\int_{\Omega}\left|w_{n} \psi^{\frac{1}{p-1}}\right|^{p \frac{\left[(p-1) m^{*}\right]^{*}}{p^{*}}}\right)^{\frac{p^{*}}{p}}+\|f \psi\|_{L^{m}(\Omega)}^{\frac{\left[(p-1) m^{*}\right]^{*}}{p-1}}\right] .
\end{aligned}
$$

Thanks to (2.11), the right-hand side of (2.25) is uniformly bounded with respect to $n$. Therefore, it follows that the sequence $\left\{w_{n} \psi^{\eta_{1}}\right\}$ is bounded in $L^{\left[(p-1) m^{*}\right]^{*}}(\Omega)$, where

$$
\eta_{1}=\frac{1}{p-1}+\frac{p^{*}}{\left[(p-1) m^{*}\right]^{*}} .
$$

If $p \frac{\left[(p-1) m^{*}\right]^{*}}{p^{*}} \geq \frac{p^{*}}{p^{\prime}}$, we perform an iteration argument. The idea is to start from estimate $(2.23)$ with $\gamma=\frac{\left[(p-1) m^{*}\right]^{*}}{p^{*}}$ and $\phi$ a suitable power of $\psi$ and apply (2.23) recursively a finite number of times, choosing $\gamma$ and $\phi$ in a suitable way. We point out that, by (2.24), it is necessary to consider only values of $\gamma>\frac{1}{p^{\prime}}$.

We define $\gamma_{0}=\frac{\left[(p-1) m^{*}\right]^{*}}{p^{*}}$ and we choose $\frac{p \gamma_{0}}{p^{*}}<\gamma_{1}<\gamma_{0}$. Notice that $\frac{p \gamma_{0}}{p^{*}} \geq \frac{1}{p^{\prime}}$, therefore $\gamma_{1}>\frac{1}{p^{\prime}}$. If $p \gamma_{1} \geq \frac{p^{*}}{p^{\prime}}$, we choose $\frac{p \gamma_{1}}{p^{*}}<\gamma_{2}<\frac{p \gamma_{0}}{p^{*}}$, which, in particular, satisfies $\frac{1}{p^{\prime}} \leq \frac{p \gamma_{1}}{p^{*}}<\gamma_{2}<\frac{p \gamma_{0}}{p^{*}}<\gamma_{1}$. The process terminates in a finite number of steps, otherwise there would be an infinite sequence of real numbers $\gamma_{j}>\gamma_{j+1}>\frac{1}{p^{\prime}}$ such that ${ }^{5} \gamma_{j}<\left(\frac{p}{p^{*}}\right)^{\left[\frac{j}{2}\right]} \gamma_{0}$ for every $j \in \mathbb{N}$, which is a contradiction, since $\left(\frac{p}{p^{*}}\right)^{\left[\frac{j}{2}\right]} \rightarrow 0$ as $j \rightarrow \infty$. If $I \geq 1$ is the first index for which

we define

$$
p \gamma_{I}<\frac{p^{*}}{p^{\prime}}
$$

$$
\phi_{I}=\psi, \quad \phi_{i}=\phi_{i+1}^{1+\frac{p-1}{\gamma_{i+1}}} \quad i=0, \ldots, I-1
$$

${ }^{5}$ For every $t \in \mathbb{R},[t]$ denotes the integer part of $t$. 
By construction, we have that $\frac{1}{p^{\prime}}<\gamma_{I}<\gamma_{I-1} \leq \ldots \leq \gamma_{0}=\frac{\left[(p-1) m^{*}\right]^{*}}{p^{*}}$ and $0 \leq \phi_{0} \leq \phi_{1} \leq \ldots \leq \phi_{I}=\psi$ in $\Omega$. Hence, we set $C_{14}=\max \left\{C_{11}\left(\gamma_{i}\right), i=\right.$ $0, \ldots, I\}=C_{11}\left(\gamma_{I}\right)$ and we consider estimate (2.23) with $\gamma=\gamma_{0}$ and $\phi=\phi_{0}$ :

$$
\int_{\Omega}\left|w_{n} \phi_{0}^{\frac{1}{p-1}+\frac{1}{\gamma_{0}}}\right|^{p^{*} \gamma_{0}} \leq C_{14}\left[\left(\int_{\Omega}\left|w_{n} \phi_{0}^{\frac{1}{p-1}}\right|^{p \gamma_{0}}\right)^{\frac{p^{*}}{p}}+\left\|f \phi_{0}\right\|_{L^{m}(\Omega)}^{\frac{p^{*} \gamma_{0}}{p-1}}\right] .
$$

Since $p \gamma_{0}<p^{*} \gamma_{1}$, by Hölder inequality and the definition of $\phi_{i}$, we have that

$$
\begin{aligned}
\left(\int_{\Omega}\left|w_{n} \phi_{0}^{\frac{1}{p-1}}\right|^{p \gamma_{0}}\right)^{\frac{p^{*}}{p}} \leq|\Omega|^{\frac{p^{*}}{p}-\frac{\gamma_{0}}{\gamma_{1}}} & \left(\int_{\Omega}\left|w_{n} \phi_{0}^{\frac{1}{p-1}}\right|^{p^{*} \gamma_{1}}\right)^{\frac{\gamma_{0}}{\gamma_{1}}} \\
& =|\Omega|^{\frac{p^{*}}{p}-\frac{\gamma_{0}}{\gamma_{1}}}\left(\int_{\Omega}\left|w_{n} \phi_{1}^{\frac{1}{p-1}+\frac{1}{\gamma_{1}}}\right|^{p^{*} \gamma_{1}}\right)^{\frac{\gamma_{0}}{\gamma_{1}}},
\end{aligned}
$$

which in turn, using (2.23), implies that

$$
\begin{aligned}
& \left(\int_{\Omega}\left|w_{n} \phi_{0}^{\frac{1}{p-1}}\right|^{p \gamma_{0}}\right)^{\frac{p^{*}}{p}} \\
& \quad \leq\left(2 C_{14}\right)^{\frac{\gamma_{0}}{\gamma_{1}}}|\Omega|^{\frac{p^{*}}{p}-\frac{\gamma_{0}}{\gamma_{1}}}\left[\left(\int_{\Omega}\left|w_{n} \phi_{1}^{\frac{1}{p-1}}\right|^{p \gamma_{1}}\right)^{\frac{p^{*} \gamma_{0}}{p \gamma_{1}}}+\left\|f \phi_{1}\right\|_{L^{m}(\Omega)}^{\frac{p^{*} \gamma_{0}}{p-1}}\right] .
\end{aligned}
$$

Putting together (2.28) and (2.29), it follows that

$$
\begin{aligned}
& \int_{\Omega}\left|w_{n} \phi_{0}^{\frac{1}{p-1}+\frac{1}{\gamma_{0}}}\right|^{p^{*} \gamma_{0}}
\end{aligned}
$$

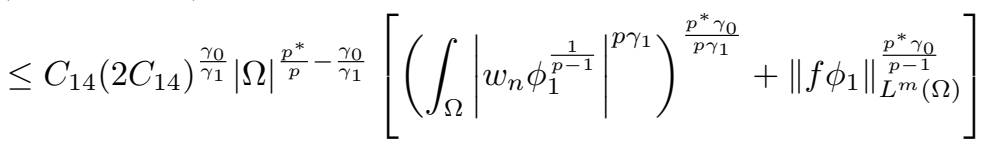

$$
\begin{aligned}
& +C_{14}\left\|f \phi_{0}\right\|_{L^{m}(\Omega)}^{\frac{p^{*} \gamma_{0}}{p-1}} .
\end{aligned}
$$

Thus, we iterate the previous inequality $I$ times and we obtain that

$$
\begin{aligned}
\int_{\Omega}\left|w_{n} \phi_{0}^{\frac{1}{p-1}+\frac{1}{\gamma_{0}}}\right|^{p^{*} \gamma_{0}} & \\
\leq & C_{15}\left[\left(\int_{\Omega}\left|w_{n} \phi_{I}^{\frac{1}{p-1}}\right|^{p \gamma_{I}}\right)^{\frac{p^{*} \gamma_{0}}{p \gamma_{I}}}+\sum_{i=0}^{I}\left\|f \phi_{i}\right\|_{L^{m}(\Omega)}^{\frac{p^{*} \gamma_{0}}{p-1}}\right]
\end{aligned}
$$

where

$$
C_{15}=C_{14}+C_{14}\left(2 C_{14}\right)^{\sum_{i=1}^{I} \frac{\gamma_{0}}{\gamma_{i}}}|\Omega|^{\sum_{i=0}^{I}\left(\frac{p^{*} \gamma_{0}}{p \gamma_{i}}-\frac{\gamma_{0}}{\gamma_{i+1}}\right)} .
$$


By (2.26) and (2.11), the right-hand side of (2.30) is uniformly bounded with respect to $n$. Therefore, since $p^{*} \gamma_{0}=\left[(p-1) m^{*}\right]^{*}$ and

$$
\begin{aligned}
\phi_{0}^{\frac{1}{p-1}+\frac{1}{\gamma_{0}}}=\phi_{1}^{\frac{1}{p-1}\left(1+\frac{p-1}{\gamma_{0}}\right)} & \left(1+\frac{p-1}{\gamma_{1}}\right) \\
& =\ldots=\phi_{I}^{\frac{1}{p-1} \prod_{i=0}^{I}\left(1+\frac{p-1}{\gamma_{i}}\right)}=\psi^{\frac{1}{p-1} \prod_{i=0}^{I}\left(1+\frac{p-1}{\gamma_{i}}\right),}
\end{aligned}
$$

from $(2.30)$ we, finally, deduce that $\left\{w_{n} \psi^{\eta_{1}}\right\}$ is bounded in $L^{\left[(p-1) m^{*}\right]^{*}}(\Omega)$, where

$$
\eta_{1}=\frac{1}{p-1} \prod_{i=0}^{I}\left(1+\frac{p-1}{\gamma_{i}}\right) .
$$

In the proof of the next local estimate on $w_{n}$ we follows Stampacchia's method (see[21]), which hinges on the following Real Analysis result (see [19] and $[20]$ for the proof).

Lemma 2.3. Let $g(\sigma)$ be a nonnegative, nonincresing function defined for every $\sigma \geq \sigma_{0}$ and such that

$$
\exists \gamma, \delta, C>0: \quad g(\tau) \leq \frac{C g(\sigma)^{\delta}}{(\tau-\sigma)^{\gamma}} \quad \forall \tau>\sigma \geq \sigma_{0} .
$$

Then

i) if $\delta>1$, it holds that

$$
g\left(\sigma_{0}+\tau_{0}\right)=0
$$

where $\tau_{0}^{\gamma}=2^{\frac{\gamma \delta}{\delta-1}} C g\left(\sigma_{0}\right)^{\delta-1} ;$

ii) if $\delta=1$, it holds that

$$
g(\sigma) \leq \frac{g\left(\sigma_{0}\right)}{e^{\zeta_{0}\left(\sigma-\sigma_{0}\right)-1}} \quad \forall \sigma \geq \sigma_{0},
$$

where $\zeta_{0}=(e C)^{-\frac{1}{\gamma}}$;

iii) if $\delta<1$ and $\sigma_{0}>0$, it holds that

$$
g(\sigma) \leq \frac{2^{\frac{\mu}{1-\delta}}\left[C^{\frac{1}{1-\delta}}+\left(2 \sigma_{0}\right)^{\mu} g\left(\sigma_{0}\right)\right]}{\sigma^{\mu}} \quad \forall \sigma \geq \sigma_{0},
$$

where $\mu=\frac{\gamma}{1-\delta}$.

In what follows, for any $\sigma>0, G_{\sigma}$ denotes the real function of one real variable defined by

$$
G_{\sigma}(s)=s-T_{\sigma}(s) \quad \forall s \in \mathbb{R},
$$

where $T_{\sigma}$ is the truncation at level $\sigma$ defined in (2.5). Furthermore, for any $n \in \mathbb{N}$ and $\eta, \sigma>0$ we define

$$
A_{n, \eta, \sigma}=\left\{x \in \Omega:\left|w_{n}(x)\right| \psi(x)^{\eta}>\sigma\right\} .
$$


Notice that, by (2.11), $\left|A_{n, \eta, \sigma}\right| \rightarrow 0$ as $\sigma \rightarrow \infty$, uniformly with respect to $n$. Hence, by the absolute continuity of the integral, it follows that

$$
\lim _{\sigma \rightarrow \infty}\|b\|_{L^{N}\left(A_{n, \eta, \sigma}\right)}=0 \text { uniformly with respect to } n \text {. }
$$

Lemma 2.4. Assume that (1.2), (1.3) with $b \in L^{N, 1}(\Omega)$, (1.8), (1.9) and (2.10) with $m>\frac{N}{p}$. Then, there exists $\eta_{2}>1$ which depends only on $\psi, N$ and $p$, such that the sequence $\left\{w_{n} \psi^{\eta_{2}}\right\}$ is bounded in $L^{\infty}(\Omega)$.

Proof. We follow the approach of [21] (see also [8]). First, we observe that, since

$$
\lim _{t \rightarrow \frac{N}{p}}\left[(p-1) t^{*}\right]^{*}=\lim _{t \rightarrow \frac{N}{p}} \frac{N(p-1) t}{N-p t}=\infty,
$$

for every $s \geq \frac{p^{*}}{p^{\prime}}$ there exists $1 \leq t<\frac{N}{p}$ such that $s=\left[(p-1) t^{*}\right]^{*}$. Therefore, by Lemma 2.2 , for every $s \geq \frac{p^{*}}{p^{\prime}}$ there exists $\eta_{1}(s)>\frac{1}{p-1}$ such that $\left\{w_{n} \psi^{\eta_{1}}\right\}$ is bounded in $L^{s}(\Omega)$.

We fix $s>\max \left\{\frac{p^{*}}{p^{\prime}}, N\right\}$ and we choose

$$
\eta_{2}=1+\eta_{1}(s) \text {. }
$$

Moreover, we define

$$
A_{\sigma}=A_{n, \eta_{2}, \sigma} \quad \forall \sigma>0, \forall n \in \mathbb{N},
$$

and, exploiting (2.34) and (2.35), we choose $\sigma_{0}>0$ such that

$$
\left\{\begin{array}{l}
\left|A_{\sigma}\right| \leq 1, \\
\mathcal{S}_{0}\left(1+\frac{\eta_{2}}{p^{\prime}}\right)\|b\|_{L^{N}\left(A_{\sigma}\right)} \leq \frac{\alpha}{2 p}, \\
\forall \sigma \geq \sigma_{0}, \forall n \in \mathbb{N} .
\end{array}\right.
$$

By (1.2), (2.6) and (1.3), the use of $v=G_{\sigma}\left(w_{n} \psi^{\eta_{2}}\right) \psi^{(p-1) \eta_{2}}$ as a test function in (2.7) yields

$$
\begin{aligned}
\alpha\left\|\left|D w_{n}\right| \psi^{\eta_{2}}\right\|_{L^{p}\left(A_{\sigma}\right)}^{p} \leq \beta \eta_{2} p \int_{A_{\sigma}}\left|D w_{n}\right|^{p-1}\left|D \psi \| w_{n}\right| \psi^{p \eta_{2}-1} & \\
& \quad+\int_{\Omega}|b(x)|\left|D w_{n}\right|^{p-1}|v|+\int_{\Omega}|f(x)||v|,
\end{aligned}
$$

which in turn, using Young inequality, implies that

$$
\begin{aligned}
\frac{\alpha}{p}\left\|\left|D w_{n}\right| \psi^{\eta_{2}}\right\|_{L^{p}\left(A_{\sigma}\right)}^{p} \leq & \frac{\left(\beta \eta_{2} p\right)^{p}}{p \alpha^{p-1}}\left\||D \psi| w_{n} \psi^{\eta_{2}-1}\right\|_{L^{p}\left(A_{\sigma}\right)}^{p} \\
& \quad+\int_{\Omega}|b(x)|\left|D w_{n}\right|^{p-1}|v|+\int_{\Omega}|f(x)||v| .
\end{aligned}
$$

Thanks to Hölder and Sobolev inequalities, the second integral on the righthand side of (2.38) can be estimated as follows

$$
\int_{\Omega}|b(x)|\left|D w_{n}\right|^{p-1}|v| \leq \mathcal{S}_{0}\|b\|_{L^{N}\left(A_{\sigma}\right)}\left\|\left|D w_{n}\right| \psi^{\eta_{2}}\right\|_{L^{p}\left(A_{\sigma}\right)}^{p-1}\left\|D\left(w_{n} \psi^{\eta_{2}}\right) \mid\right\|_{L^{p}\left(A_{\sigma}\right)} .
$$


Hence, using Young inequality again and (2.37), we get

$$
\begin{aligned}
\int_{\Omega}|b(x)|\left|D w_{n}\right|^{p-1}|v| \leq & \frac{\alpha}{2 p} \\
& \left\|\left|D w_{n}\right| \psi^{\eta_{2}}\right\|_{L^{p}\left(A_{\sigma}\right)}^{p} \\
& +\frac{\mathcal{S}_{0} \eta_{2}\|b\|_{L^{N}(\Omega)}}{p}\left\||D \psi| w_{n} \psi^{\eta_{2}-1}\right\|_{L^{p}\left(A_{\sigma}\right)}^{p}
\end{aligned}
$$

Putting together (2.38) and (2.39), it follows that

$$
C_{0}\left\|\left|D w_{n}\right| \psi^{\eta_{2}}\right\|_{L^{p}\left(A_{\sigma}\right)}^{p} \leq C_{1}\left\||D \psi| w_{n} \psi^{\eta_{2}-1}\right\|_{L^{p}\left(A_{\sigma}\right)}^{p}+\int_{\Omega}|f(x) \| v|,
$$

where $C_{0}=\frac{\alpha}{2 p}$ and $C_{1}=\frac{\left(\beta \eta_{2} p\right)^{p}}{p \alpha^{p-1}}+\frac{\mathcal{S}_{0} \eta_{2}\|b\|_{L^{N}(\Omega)}}{p}$. Adding $C_{0}\left\|\left|D \psi^{\eta_{2}}\right| w_{n}\right\|_{L^{p}\left(A_{\sigma}\right)}^{p}$ on both sides of (2.40) and using Sobolev inequality again, we obtain that

$$
\begin{array}{r}
C_{2}\left\|G_{\sigma}\left(w_{n} \psi^{\eta_{2}}\right)\right\|_{L^{p^{*}}(\Omega)}^{p} \leq C_{0}\left\|\left|D w_{n}\right| \psi^{\eta_{2}}\right\|_{L^{p}\left(A_{\sigma}\right)}^{p}+C_{0}\left\|\left|D \psi^{\eta_{2}}\right| w_{n}\right\|_{L^{p}\left(A_{\sigma}\right)}^{p} \\
\leq C_{3}\left\|w_{n} \psi^{\eta_{2}-1}\right\|_{L^{p}\left(A_{\sigma}\right)}^{p}+\int_{\Omega}|f(x) \| v|, \quad
\end{array}
$$

where $C_{2}=\frac{C_{0}}{\mathcal{S}_{0}^{p}}$ and $C_{3}=C_{1}+C_{0}\left(\eta_{2}\|\mid D \psi\|_{L^{\infty}(\Omega)}\right)^{p}$.

Since $\sigma>\max \left\{\frac{p^{*}}{p^{\prime}}, N\right\} \geq N>p$, Hölder inequality implies that

$$
\left\|w_{n} \psi^{\eta_{2}-1}\right\|_{L^{p}\left(A_{\sigma}\right)}^{p} \leq\left\|w_{n} \psi^{\eta_{2}-1}\right\|_{L^{s}(\Omega)}^{p}\left|A_{\sigma}\right|^{1-\frac{p}{s}}
$$

which in turn, recalling that $\eta_{2}=1+\eta_{1}$ and $\left\{w_{n} \psi^{\eta_{1}}\right\}$ is bounded in $L^{s}(\Omega)$, yields

$$
\left\|w_{n} \psi^{\eta_{1}}\right\|_{L^{p}\left(A_{\sigma}\right)}^{p} \leq C_{4}\left|A_{\sigma}\right|^{1-\frac{p}{s}}
$$

On the other hand, by Hölder and Young inequalities, we have that

$$
\begin{aligned}
& \int_{\Omega}\left|f(x)\left\|v \mid \leq \frac{c_{2}}{p}\right\| G_{\sigma}\left(w_{n} \psi^{\eta_{2}}\right) \|_{L^{p^{*}}(\Omega)}^{p}+\frac{1}{p^{\prime} C_{2}^{\frac{1}{p-1}}\|f \psi\|_{L^{\left(p^{*}\right)^{\prime}}\left(A_{\sigma}\right)}^{p^{\prime}}}\right. \\
& \leq \frac{C_{2}}{p}\left\|G_{\sigma}\left(w_{n} \psi^{\eta_{2}}\right)\right\|_{L^{p^{*}}(\Omega)}^{p}+\frac{1}{p^{\prime} C_{2}^{\frac{1}{p-1}}\|f \psi\|_{L^{m}(\Omega)}^{p^{\prime}}\left|A_{\sigma}\right|^{p^{\prime}\left(\frac{1}{m^{\prime}}-\frac{1}{p^{*}}\right)}}
\end{aligned}
$$

Putting together (2.41)-(2.43) it follows that

$$
C_{5}\left\|G_{\sigma}\left(w_{n} \psi^{\eta_{2}}\right)\right\|_{L^{p^{*}}(\Omega)}^{p} \leq C_{6}\left|A_{\sigma}\right|^{1-\frac{p}{s}}+C_{7}\left|A_{\sigma}\right|^{p^{\prime}\left(\frac{1}{m^{\prime}}-\frac{1}{p^{*}}\right)},
$$

where $C_{5}=\frac{C_{2}}{p^{\prime}}, C_{6}=C_{3} C_{4}$ and $C_{7}=\frac{1}{p^{\prime} C_{2}^{\frac{1}{p-1}}}\|f \psi\|_{L^{m}(\Omega)}^{p^{\prime}}$.

Then, we have that

$$
\begin{aligned}
& C_{5}(\tau-\sigma)^{p}\left|A_{\tau}\right|^{\frac{p}{p^{*}}} \leq C_{5}\left\|G_{\sigma}\left(w_{n} \psi^{\eta_{2}}\right)\right\|_{L^{p^{*}}(\Omega)}^{p} \\
& \quad \leq C_{6}\left|A_{\sigma}\right|^{1-\frac{p}{s}}+C_{7}\left|A_{\sigma}\right|^{p^{\prime}\left(\frac{1}{m^{\prime}}-\frac{1}{p^{*}}\right)} \quad \forall \tau>\sigma \geq \sigma_{0} .
\end{aligned}
$$

Since $s>N$ and $m>\frac{N}{p}$, it holds that $1-\frac{p}{s}>p^{\prime}\left(\frac{1}{m^{\prime}}-\frac{1}{p^{*}}\right)$. Hence, recalling that $\left|A_{\sigma}\right|<1$, we obtain that

$$
(\tau-\sigma)^{p}\left|A_{\tau}\right|^{\frac{p}{p^{*}}} \leq C_{8}\left|A_{\sigma}\right|^{p^{\prime}\left(\frac{1}{m^{\prime}}-\frac{1}{p^{*}}\right)} \quad \forall \tau>\sigma \geq \sigma_{0},
$$


that is

$$
\left|A_{\tau}\right| \leq C_{9} \frac{\left|A_{\sigma}\right|^{\frac{1}{p-1}\left(\frac{p^{*}}{m^{\prime}}-1\right)}}{(\tau-\sigma)^{p^{*}}} \quad \forall \tau>\sigma \geq \sigma_{0},
$$

where $C_{8}=\frac{C_{6}+C_{7}}{C_{5}}$ and $C_{9}=C_{8}^{\frac{p^{*}}{p}}$.

Now, assumption $m>\frac{N}{p}$ implies that $\frac{1}{p-1}\left(\frac{p^{*}}{m^{\prime}}-1\right)>1$. Thus, applying Lemma 2.3 (part i)) with $g(\sigma)=\left|A_{\sigma}\right|$, from (2.44) we finally deduce that there exists $\tau_{0}>0$ (not depending on $n$ ) such that $\left|A_{\sigma_{0}+\tau_{0}}\right|=0$, that is, $\left|w_{n} \psi^{\eta_{2}}\right| \leq \sigma_{0}+\tau_{0}$ a.e. in $\Omega$.

Remark 2.1. We observe that in order to get inequality (2.44), assumption (2.10) with $m>\frac{N}{p}$ is not needed, but it is sufficient to assume the weaker condition

$$
\left\{\begin{array}{l}
\exists \psi \in W^{1, \infty}(\Omega), m>\left(p^{*}\right)^{\prime}: \\
0 \leq \psi \leq 1 \quad \text { in } \Omega, \\
f \psi \in M^{m}(\Omega) .
\end{array}\right.
$$

As a matter of fact, (2.45) implies that $f \psi \in L^{t}(\Omega)$ for every $\left(p^{*}\right)^{\prime} \leq t<m$. Hence, by Lemma 2.2, setting

$$
s_{0}= \begin{cases}\infty & \text { if } m \geq \frac{N}{p}, \\ {\left[(p-1) m^{*}\right]^{*}} & \text { if }\left(p^{*}\right)^{\prime}<m<\frac{N}{p},\end{cases}
$$

for every $\frac{p^{*}}{p^{\prime}} \leq s<s_{0}$ there exists $\eta_{1}(s)>\frac{1}{p-1}$ such that $\left\{w_{n} \psi^{\eta_{1}}\right\}$ is bounded in $L^{s}(\Omega)$. Moreover, it holds that

$$
\exists C>0: \quad \int_{U}|f \psi|^{\left(p^{*}\right)^{\prime}} \leq C|U|^{1-\frac{\left(p^{*}\right)^{\prime}}{m}} \quad \forall \text { measurable subset } U \subset \Omega .
$$

In particular, we have that

$$
\|f \psi\|_{L^{\left(p^{*}\right)^{\prime}\left(A_{n, \eta, \sigma}\right)}}^{p^{\prime}} \leq C^{\frac{p^{\prime}}{\left(p^{*}\right)^{\prime}}}\left|A_{n, \eta, \sigma}\right|^{p^{\prime}\left(\frac{1}{m^{\prime}}-\frac{1}{p^{*}}\right)} \quad \forall \eta, \sigma>0, \forall n \in \mathbb{N},
$$

where $A_{n, \eta, \sigma}$ is defined in (2.34). Hence, the same argument of the proof of Lemma 2.4 can be used also in this case to deduce inequality (2.44). The only things that change are the choice of $s$ and the use of (2.46) instead of Hölder inequality in (2.43).

Therefore, applying Lemma 2.3 with $g(\sigma)=\left|A_{n, \eta_{2}, \sigma}\right|, \gamma=p^{*}$ and $\delta=$ $\frac{1}{p-1}\left(\frac{1}{m^{\prime}}-\frac{1}{p^{*}}\right)$, we immediately obtain that:

- if (2.45) holds with $m>\frac{N}{p}$, then $\left\{w_{n} \psi^{\eta_{2}}\right\}$ is bounded in $L^{\infty}(\Omega)$;

- if (2.45) holds with $\left(p^{*}\right)^{\prime}<m<\frac{N}{p}$, then $\left\{w_{n} \psi^{\eta_{2}}\right\}$ is bounded in $M^{\left[(p-1) m^{*}\right]^{*}}(\Omega)$.

Moreover, if (2.45) is fulfilled with $m=\frac{N}{p}$, which, in particular, implies that $\frac{1}{p-1}\left(\frac{p^{*}}{m^{\prime}}-1\right)=1$, thanks to $(2.44)$ and Lemma 2.3 (part ii)), it follows that

$$
\left|A_{n, \eta_{2}, \sigma}\right| \leq \frac{\left|A_{n, \eta_{2}, \sigma_{0}}\right|}{e^{\zeta_{0}\left(\sigma-\sigma_{0}\right)-1}} \leq \frac{|\Omega|}{e^{\zeta_{0}\left(\sigma-\sigma_{0}\right)-1}} \quad \forall \sigma \geq \sigma_{0},
$$


where $\zeta_{0}=\left(e C_{9}\right)^{-\frac{1}{p^{*}}}$. Consequently, by a standard device, we deduce that

$$
\int_{\Omega}\left(e^{\zeta\left|G_{\sigma_{0}}\left(w_{n} \psi^{\eta_{2}}\right)\right|}-1\right) \leq \frac{e \zeta|\Omega|}{\zeta_{0}-\zeta} \quad \forall 0<\zeta<\zeta_{0},
$$

which implies that the sequence $\left\{e^{\zeta\left|w_{n}\right| \psi^{\eta_{2}}}\right\}$ is bounded in $L^{1}(\Omega)$.

Recalling that $w_{n} \rightarrow w$ and $D w_{n} \rightarrow D w$ a.e. in $\Omega$ with $w$ weak solution to (1.4), the previous estimates, together with those given in Lemmas 2.6 and 2.7 below, lead to the following result.

Theorem 2.5. Assume (1.2), (1.3) with $b \in L^{N, 1}(\Omega)$, (1.8), (1.9) and (2.45) with $m>\left(p^{*}\right)^{\prime}$. Then, there exist a weak solution $w$ to $(1.4)$ and $\eta_{0}>1$ which depends only on $\psi, m, N$ and $p$, such that

$$
w \psi^{\eta_{0}} \in W_{0}^{1, p}(\Omega)
$$

and

$$
\begin{cases}w \psi^{\eta_{0}} \in L^{\infty}(\Omega) & \text { if } m>\frac{N}{p}, \\ e^{\zeta|w| \psi^{\eta_{0}} \in L^{1}(\Omega) \quad \text { for some } \zeta>0} & \text { if } m=\frac{N}{p}, \\ w \psi^{\eta_{0}} \in M^{\left[(p-1) m^{*}\right]^{*}}(\Omega) & \text { if }\left(p^{*}\right)^{\prime}<m<\frac{N}{p} .\end{cases}
$$

\subsection{Local estimates on $D w_{n}$}

The next two lemmas provide estimates on the sequence $\left\{\left|D\left(w_{n} \psi^{\eta}\right)\right|\right\}$ for some $\eta>1$.

Lemma 2.6. Assume (1.2), (1.3) with $b \in L^{N, 1}(\Omega)$, (1.8), (1.9) and (2.10) with $1<m<\left(p^{*}\right)^{\prime}$. Then, there exists $\eta_{3}>1$ which depends only on $\psi, m$, $N$ and $p$, such that the sequence $\left\{\left|D w_{n}\right|^{(p-1) m^{*}} \psi^{\eta_{3}}\right\}$ is bounded in $L^{1}(\Omega)$.

Proof. We define $q=(p-1) m^{*}, \gamma=\frac{q^{*}}{p^{*}}$ and

$$
\eta_{3}=\max \left\{\frac{q^{*} \eta_{1}}{m^{\prime}}+1, \frac{p q^{*} \eta_{1}}{p^{*}}+p, q^{*} \eta_{1}\right\},
$$

where $\eta_{1}$ is given by Lemma 2.2. Notice that $1<m<\left(p^{*}\right)^{\prime}$ implies that $\frac{1}{p^{\prime}}<\gamma<1$. Moreover, we have that $[1-p(1-\gamma)] m^{\prime}=p^{*} \gamma=q^{*}$. Then, we fix $\delta>0$ and we choose

$$
\left[\left(\delta+\left|w_{n}\right|\right)^{1-p(1-\gamma)}-\delta^{1-p(1-\gamma)}\right] \operatorname{sign}\left(w_{n}\right) \psi^{\eta_{3}}
$$

as a test function in (2.7). Arguing as in the first part of the proof of Lemma 2.2 (see $(2.20))$, we get

$$
\begin{aligned}
& \int_{\Omega} \frac{\left|D w_{n}\right|^{p}}{\left(\delta+\left|w_{n}\right|\right)^{p(1-\gamma)}} \psi^{\eta_{3}} \\
& \quad \leq C_{0} \int_{\Omega}\left(\delta+\left|w_{n}\right|\right)^{p \gamma} \psi^{\eta_{3}-p}+C_{1} \int_{\Omega}|f(x)|\left(\delta+\left|w_{n}\right|\right)^{p(\gamma-1)+1} \psi^{\eta_{3}}
\end{aligned}
$$

where $C_{1}$ and $C_{1}$ are positive constants that do not depend on $n$. By Hölder inequality, we have that

$$
C_{0} \int_{\Omega}\left(\delta+\left|w_{n}\right|\right)^{p \gamma} \psi^{\eta_{3}-p} \leq C_{0}|\Omega|^{1-\frac{p}{p^{*}}}\left[\int_{\Omega}\left(\delta+\left|w_{n}\right|\right)^{q^{*}} \psi^{\frac{p^{*}\left(\eta_{3}-p\right)}{p}}\right]^{\frac{p}{p^{*}}},
$$


and

$$
\begin{aligned}
C_{1} \int_{\Omega}|f(x)|\left(\delta+\left|w_{n}\right|\right)^{p(\gamma-1)+1} \psi^{\eta_{3}} & \\
& \leq C_{1}\|f \psi\|_{L^{m}(\Omega)}\left[\int_{\Omega}\left(\delta+\left|w_{n}\right|\right)^{q^{*}} \psi^{\left(\eta_{3}-1\right) m^{\prime}}\right]^{\frac{1}{m^{\prime}}}
\end{aligned}
$$

which in turn, recalling the definitions of $q$ and $\eta_{3}$ and the fact that $0 \leq \psi \leq 1$ in $\Omega$, imply that

$$
C_{0} \int_{\Omega}\left(\delta+\left|w_{n}\right|\right)^{p \gamma} \psi^{\eta_{3}-p} \leq C_{0}|\Omega|^{1-\frac{p}{p^{*}}}\left\|\left(\delta+\left|w_{n}\right|\right) \psi^{\eta_{1}}\right\|_{L^{\left[(p-1) m^{*}\right]^{*}}(\Omega)}^{\frac{p\left[(p-1) m^{*}\right]^{*}}{p^{*}},}
$$

and

$$
\begin{aligned}
C_{1} \int_{\Omega}|f(x)|\left(\delta+\left|w_{n}\right|\right)^{p(\gamma-1)+1} \psi^{\eta_{3}} & \\
& \leq C_{1}\|f \psi\|_{L^{m}(\Omega)}\left\|\left(\delta+\left|w_{n}\right|\right) \psi^{\eta_{1}}\right\|_{L^{\left[(p-1) m^{*}\right]^{*}}(\Omega)}^{\frac{\left[(p-1) m^{*}\right]^{*}}{m^{\prime}}} .
\end{aligned}
$$

Hence, putting together (2.49)-(2.51), by Lemma 2.2, it follows that the quantity

$$
\int_{\Omega} \frac{\left|D w_{n}\right|^{p}}{\left(\delta+\left|w_{n}\right|\right)^{p(1-\gamma)}} \psi^{\eta_{3}}
$$

is uniformly bounded with respect to $n$.

Now, using Hölder inequality again, we have that

$$
\begin{aligned}
\int_{\Omega}\left|D w_{n}\right|^{q} & \psi^{\eta_{3}} \leq \int_{\Omega} \frac{\left|D w_{n}\right|^{p}}{\left(\delta+\left|w_{n}\right|\right)^{p(1-\gamma)}}\left(\delta+\left|w_{n}\right|\right)^{q(1-\gamma)} \psi^{\eta_{3}} \\
\leq & {\left[\int_{\Omega} \frac{\left|D w_{n}\right|^{p}}{\left(\delta+\left|w_{n}\right|\right)^{p(1-\gamma)}} \psi^{\eta_{3}}\right]^{\frac{p}{q^{*}}}\left[\int_{\Omega}\left(\delta+\left|w_{n}\right|\right)^{\frac{p q(1-\gamma)}{p-q}} \psi^{\eta_{3}}\right]^{1-\frac{p}{q^{*}}} . }
\end{aligned}
$$

A simple calculation shows that $\frac{p q(1-\gamma)}{p-q}=q^{*}=\left[(p-1) m^{*}\right]^{*}$. Therefore, recalling the choice of $\eta_{3}$ and the fact that $0 \leq \psi \leq 1$ in $\Omega$, thanks to Lemma 2.2 , from the previous inequality we finally deduce the result.

Lemma 2.7. Assume that (1.2), (1.3) with $b \in L^{N, 1}(\Omega),(1.8)$, (1.9) and (2.10) with $m=\left(p^{*}\right)^{\prime}$. Then, there exists $\eta_{4}>1$ which depends only on $\psi, N$ and $p$, such that the sequence $\left\{\left|D w_{n}\right|^{p} \psi^{\eta_{4}}\right\}$ is bounded in $L^{1}(\Omega)$.

Proof. We define

$$
\eta_{4}=p\left(1+\eta_{1}\right)
$$

where $\eta_{1}$ is given by Lemma 2.2 above, and we choose $w_{n} \psi^{\eta_{4}}$ as a test function in (2.7). Arguing as in the first part of the proof of Lemma 2.2 (see (2.20)), we obtain that

$$
\int_{\Omega}\left|D w_{n}\right|^{p} \psi^{\eta_{4}} \leq C_{0} \int_{\Omega}\left|w_{n}\right|^{p} \psi^{\eta_{4}-p}+C_{1} \int_{\Omega}|f(x)|\left|w_{n}\right| \psi^{\eta_{4}},
$$


where $C_{0}$ and $C_{1}$ are positive constants that do not depend on $n$. Now, the choice of $\eta_{4}$ implies that

$$
C_{0} \int_{\Omega}\left|w_{n}\right|^{p} \psi^{\eta_{4}-p}=C_{0}\left\|w_{n} \psi^{\eta_{1}}\right\|_{L^{p}(\Omega)}^{p} .
$$

Moreover, the use Hölder inequality and the fact that $0 \leq \psi \leq 1$ in $\Omega$ lead to

$$
C_{1} \int_{\Omega}|f(x)|\left|w_{n}\right| \psi^{\eta_{4}} \leq C_{1}\|f \psi\|_{L^{\left(p^{*}\right)^{\prime}(\Omega)}}\left\|w_{n} \psi^{\eta_{1}}\right\|_{L^{p^{*}(\Omega)}} .
$$

Hence, from $(2.52),(2.53)$ and $(2.54)$ it follows that

$$
\int_{\Omega}\left|D w_{n}\right|^{p} \psi^{\eta_{4}} \leq C_{0}\left\|w_{n} \psi^{\eta_{1}}\right\|_{L^{p}(\Omega)}^{p}+C_{1}\|f \psi\|_{L^{\left(p^{*}\right)^{\prime}}(\Omega)}\left\|w_{n} \psi^{\eta_{1}}\right\|_{L^{p *}(\Omega)},
$$

which, thanks to Lemma 2.2, implies the result, since $p^{*}=\left[(p-1) m^{*}\right]^{*}$.

\subsection{Proof of Theorem 2.1}

Let $\left\{w_{n}\right\}$ be the sequence of weak solutions of the approximate problems $(2.7)$ constructed above. Closely following the outline of the proof of Theorem 2.1 in [4], we can prove that there exists a weak solution $w$ of (1.4) such that, up to a subsequence, $w_{n} \rightarrow w$ and $D w_{n} \rightarrow D w$ a.e. in $\Omega$. Therefore, the result follows immediately by Lemmas $2.2,2.4,2.6$ and 2.7 choosing $\eta_{0}$ in a suitable way.

\section{Local regularity results for (1.11)}

Following the main ideas of the previous section, here we study local regularity properties of weak solutions to (1.11) with datum in $L^{1}(\Omega)$.

Let $f \in L^{1}(\Omega)$. We recall that a function $u: \Omega \rightarrow \mathbb{R}$ is a weak solution of (1.11) if $u$ satisfies

$$
|a(\cdot, u, D u)| \in L_{\mathrm{loc}}^{1}(\Omega), \quad B(\cdot, D u) \in L_{\mathrm{loc}}^{1}(\Omega), \quad K(\cdot, u) \in L_{\mathrm{loc}}^{1}(\Omega),
$$

and

$$
\left\{\begin{array}{l}
\int_{\Omega} a(x, u, D u) \cdot D \varphi+\int_{\Omega} B(x, D u) \varphi+\int_{\Omega} K(x, u) \varphi=\int_{\Omega} f(x) \varphi, \\
\forall \varphi \in C_{c}^{\infty}(\Omega) .
\end{array}\right.
$$

The existence of a weak solution $u$ to (1.11) that satisfies (1.7) can be deduced as in [4] assuming (1.8) and that $b \in L^{N, 1}(\Omega)$, because of the coercivity properties of the zero order term $K(x, u)$. On the other hand, if $k$ satisfies (1.14), these assumptions can be weakened, as shown in [10]; moreover, there is an improvement in the regularity properties of $u$ and $D u$ with respect to (1.7).

In both cases the weak solution $u$ is obtained as limit of a sequence of regular solutions to the following family of approximate problems $(n \in \mathbb{N})$ :

$$
\begin{cases}-\operatorname{div}\left(a\left(x, u_{n}, D u_{n}\right)\right)+B_{n}\left(x, D u_{n}\right)+K\left(x, u_{n}\right)=f_{n}(x) & \text { in } \Omega \\ u_{n}=0 & \text { on } \partial \Omega\end{cases}
$$


where $B_{n}$ and $f_{n}$ are defined in (2.4). Thanks to Theorem 2.1 in [13] and Stampacchia's $L^{\infty}$-estimates (see [21]), for every $n \in \mathbb{N}$ there exists a weak solution $u_{n} \in W_{0}^{1, p}(\Omega) \cap L^{\infty}(\Omega)$ to $(3.1)$ such that $K\left(\cdot, u_{n}\right) \in L^{1}(\Omega)$ and

$$
\left\{\begin{array}{l}
\int_{\Omega} a\left(x, u_{n}, D u_{n}\right) \cdot D v+\int_{\Omega} B_{n}\left(x, D u_{n}\right) v+\int_{\Omega} K\left(x, u_{n}\right) v=\int_{\Omega} f_{n}(x) v, \\
\forall v \in W_{0}^{1, p}(\Omega) \cap L^{\infty}(\Omega) .
\end{array}\right.
$$

As already remarked in the previous section, by means of standard regularization techniques, assumptions (1.10) and (1.22) imply the following conditions:

$$
\left\{\begin{array}{l}
\exists \psi \in W^{1, \infty}(\Omega), m>1: \\
0 \leq \psi \leq 1 \quad \text { in } \Omega, \\
f \psi \in L^{m}(\Omega),
\end{array}\right.
$$

and

$$
\left\{\begin{array}{l}
\exists \psi \in W^{1, \infty}(\Omega), h>0: \\
0 \leq \psi \leq 1 \quad \text { in } \Omega, \\
k^{-h} \in L^{1}(\Omega) .
\end{array}\right.
$$

Hence, Theorems 1.2 and 1.3 are a consequence of the following results.

Theorem 3.1. Assume (1.2), (1.3) with $b \in L^{r}(\Omega)$ for some $p<r<N$, (1.9), (1.12)-(1.14) and (3.3). Assume also that $\lambda>\underline{\lambda}$ where $\underline{\lambda}$ is defined in (1.17). Then, there exist a weak solution $u$ to (1.11) and $\tilde{\eta}_{0}>1$ which depends only on $\psi, h, m, N, p$ and $r$, such that

$$
\begin{cases}u \psi^{\tilde{\eta}_{0}} \in W_{0}^{1, p}(\Omega) & \text { if } \lambda \geq \bar{\lambda} \\ u \psi^{\tilde{\eta}_{0}} \in W_{0}^{1, \tilde{q}}(\Omega) & \text { if } \underline{\lambda}<\lambda<\bar{\lambda}\end{cases}
$$

and

$$
K(\cdot, u)|u|^{\tilde{\lambda}-\lambda} \psi^{\tilde{\eta}_{0}} \in L^{1}(\Omega),
$$

where $\bar{\lambda}, \tilde{\lambda}$ and $\tilde{q}$ are defined in (1.18)-(1.20).

Theorem 3.2. Assume that (1.2), (1.3) with $b \in L^{N, 1}(\Omega)$, (1.8), (1.9), (1.12), (1.13) and (3.4). Assume also that $\lambda>\underline{\lambda}$, where $\underline{\lambda}$ is as in (1.17) but with $r=N$. Then, there exist a weak solution $u$ to (1.4) and $\tilde{\eta}_{1}>1$ which depends only on $\psi, N, p$ and $s$, such that

$$
u \psi^{\tilde{\eta}_{1}} \in W_{0}^{1, q}(\Omega) \quad \forall 1 \leq q<\tilde{q}_{1}, \quad K(\cdot, u) \psi^{\tilde{\eta}_{1}} \in L^{1}(\Omega) .
$$

Moreover, if (3.3) is fulfilled, then there exists $\tilde{\eta}_{2}>1$ which depends only on $\psi, h, m, N$ and $p$, such that

$$
\begin{cases}u \psi^{\tilde{\eta}_{2}} \in W_{0}^{1, p}(\Omega) & \text { if } \lambda \geq \bar{\lambda} \\ u \psi^{\tilde{\eta}_{2}} \in W_{0}^{1, \tilde{q}}(\Omega) & \text { if } \underline{\lambda}<\lambda<\bar{\lambda}\end{cases}
$$

and

$$
K(\cdot, u)|u|^{\tilde{\lambda}-\lambda} \psi^{\tilde{\eta}_{2}} \in L^{1}(\Omega) .
$$

where $\bar{\lambda}, \tilde{\lambda}$ and $\tilde{q}$ are as in (1.18)-(1.20) but with $r=N$. 


\subsection{Local estimates on $u_{n}$ and $D u_{n}$}

The following lemmas play the role of Lemmas 2.2, 2.4, 2.6 and 2.7 for the problem (1.11).

Lemma 3.3. Assume (1.2), (1.3) with $b \in L^{r}(\Omega)$ for some $p<r<N$, (1.9), (1.12)-(1.14) and (3.3). Assume also that $\lambda>\underline{\lambda}$, where $\underline{\lambda}$ is defined in (1.17). Then, there exists $\tilde{\eta}_{0}>1$ which depends only on $\psi, h, m, p$ and $r$, such that the sequences $\left\{\left|D u_{n}\right|{ }^{\tilde{p}} \psi^{\tilde{\eta}_{0}}\right\}$ and $\left\{K\left(\cdot, u_{n}\right)\left|u_{n}\right|^{\tilde{\lambda}-\lambda} \psi^{\tilde{\eta}_{0}}\right\}$ are bounded in $L^{1}(\Omega)$, where $\bar{\lambda}, \tilde{\lambda}$ and $\tilde{q}$ are defined in (1.18)-(1.20) above, and

$$
\tilde{p}= \begin{cases}p & \text { if } \lambda \geq \bar{\lambda} \\ \tilde{q} & \text { if } \underline{\lambda}<\lambda<\bar{\lambda} .\end{cases}
$$

Proof. We fix $\gamma>\frac{1}{p^{\prime}}, \delta>0, \eta>p$ and we choose

$$
v_{\delta} \psi^{\eta}=\left[\left(\delta+\left|u_{n}\right|\right)^{p(\gamma-1)+1}-\delta^{p(\gamma-1)+1}\right] \operatorname{sign}\left(u_{n}\right) \psi^{\eta}
$$

as a test function in (3.2). Notice that $\gamma>\frac{1}{p^{\prime}}$ implies $p(\gamma-1)+1>0$. Since

$$
D\left(v_{\delta} \psi^{\eta}\right)=[p(\gamma-1)+1] D u_{n}\left(\delta+\left|u_{n}\right|\right)^{p(\gamma-1)} \psi^{\eta}+\eta D \psi v_{\delta} \psi^{\eta-1},
$$

exploiting (1.2), (2.6) and (1.3), we obtain that

$$
\begin{aligned}
\alpha[p(\gamma-1)+1] \int_{\Omega}\left|D u_{n}\right|^{p}\left(\delta+\left|u_{n}\right|\right)^{p(\gamma-1)} \psi^{\eta} & +\int_{\Omega} K\left(x, u_{n}\right) v_{\delta} \psi^{\eta} \\
\leq \beta \eta\||D \psi|\|_{L^{\infty}(\Omega)} \int_{\Omega}\left|D u_{n}\right|^{p-1}\left|v_{\delta}\right| \psi^{\eta-1}+ & \int_{\Omega}|b(x)|\left|D u_{n}\right|^{p-1}\left|v_{\delta}\right| \psi^{\eta} \\
& +\int_{\Omega}|f(x)|\left|v_{\delta}\right| \psi^{\eta}
\end{aligned}
$$

Thanks to Young inequality, the first and second integrals on the right-hand side of (3.6) can be estimated by

$$
\begin{aligned}
\frac{\alpha[p(\gamma-1)+1]}{2 p^{\prime}} \int_{\Omega}\left|D u_{n}\right|^{p}\left(\delta+\left|u_{n}\right|\right)^{p(\gamma-1)} \psi^{\eta} & \\
+ & \frac{2^{p-1}\left(\beta \eta\||D \psi|\|_{L^{\infty}(\Omega)}\right)^{p}}{p\{\alpha[p(\gamma-1)+1]\}^{p-1}} \int_{\Omega}\left(\delta+\left|u_{n}\right|\right)^{p \gamma} \psi^{\eta-p},
\end{aligned}
$$

and

$$
\begin{aligned}
& \frac{\alpha[p(\gamma-1)+1]}{2 p^{\prime}} \int_{\Omega}\left|D u_{n}\right|^{p}\left(\delta+\left|u_{n}\right|\right)^{p(\gamma-1)} \psi^{\eta} \\
& +\frac{2^{p-1}}{p\{\alpha[p(\gamma-1)+1]\}^{p-1}} \int_{\Omega}|b(x)|^{p}\left(\delta+\left|u_{n}\right|\right)^{p \gamma} \psi^{\eta},
\end{aligned}
$$


respectively. Hence, from (3.6) we get

$$
\begin{gathered}
\int_{\Omega} K\left(x, u_{n}\right) v_{\delta} \psi^{\eta} \leq C_{0} \int_{\Omega}\left|D u_{n}\right|^{p}\left(\delta+\left|u_{n}\right|\right)^{p(\gamma-1)} \psi^{\eta}+\int_{\Omega} K\left(x, u_{n}\right) v_{\delta} \psi^{\eta} \\
\leq C_{1} \int_{\Omega}\left(\delta+\left|u_{n}\right|\right)^{p \gamma} \psi^{\eta-p}+C_{2} \int_{\Omega}|b(x)|^{p}\left(\delta+\left|u_{n}\right|\right)^{p \gamma} \psi^{\eta} \\
+\int_{\Omega}|f(x)|\left|v_{\delta}\right| \psi^{\eta}
\end{gathered}
$$

which in turn, letting $\delta \rightarrow 0$ and applying dominate convergence Theorem, implies that

$$
\begin{aligned}
& \int_{\Omega}\left|K\left(x, u_{n}\right)\right|\left|u_{n}\right|^{p(\gamma-1)+1} \psi^{\eta} \\
& \leq C_{1} \int_{\Omega}\left|u_{n}\right|^{p \gamma} \psi^{\eta-p}+C_{2} \int_{\Omega}|b(x)|^{p}\left|u_{n}\right|^{p \gamma} \psi^{\eta} \\
&+\int_{\Omega}|f(x)|\left|u_{n}\right|^{p(\gamma-1)+1} \psi^{\eta}
\end{aligned}
$$

where $C_{0}=\frac{\alpha[p(\gamma-1)+1]}{2 p}, C_{1}=\frac{2^{p-1}\left(\beta \eta\||D \psi|\|_{L^{\infty}(\Omega)}\right)^{p}}{p\{\alpha[p(\gamma-1)+1]\}^{p-1}}$ and $C_{2}=\frac{2^{p-1}}{p\{\alpha[p(\gamma-1)+1]\}^{p-1}}$. Using Hölder inequality and (1.14), we have that

$$
\begin{gathered}
C_{3} \int_{\Omega}\left|u_{n}\right|^{p \gamma} \psi^{\eta-p} \leq C_{1}|\Omega|^{\frac{p}{r}}\left\|\left|u_{n}\right|^{p \gamma} \psi^{\eta-p}\right\|_{L^{\frac{r}{r-p}}(\Omega)} \\
\leq C_{1}|\Omega|^{\frac{p}{r}}\left\|k^{-h}\right\|_{L^{1}(\Omega)}^{\frac{r-p}{(h+1) r}}\left\|k\left|u_{n}\right|^{\frac{p(h+1) r \gamma}{(r-p) h}} \psi^{\frac{(h+1) r(\eta-p)}{(r-p) h}}\right\|_{L^{1}(\Omega)}^{\frac{(r-p) h}{(h+1) r}} \\
C_{2} \int_{\Omega}|b(x)|^{p}\left|u_{n}\right|^{p \gamma} \psi^{\eta} \leq C_{2}\|b\|_{L^{r}(\Omega)}^{p}\left\|\left|u_{n}\right|^{p \gamma} \psi^{\eta}\right\|_{L^{\frac{r}{r-p}}(\Omega)} \\
\leq C_{2}\|b\|_{L^{r}(\Omega)}^{p}\left\|k^{-h}\right\|_{L^{1}(\Omega)}^{\frac{r-p}{(h+1) r}}\left\|k\left|u_{n}\right|^{\frac{p(h+1) r \gamma}{(r-p) h}} \psi^{\frac{(h+1) r \eta}{(r-p) h}}\right\|_{L^{1}(\Omega)}^{\frac{(r-p) h}{(h+1) r}}
\end{gathered}
$$

and

$$
\begin{aligned}
& \left.\int_{\Omega}\left|f(x)\left\|\left.u_{n}\right|^{p(\gamma-1)+1} \psi^{\eta} \leq\right\| f \psi\left\|_{L^{m}(\Omega)}\right\|\right| u_{n}\right|^{p(\gamma-1)+1} \psi^{\eta-1} \|_{L^{m^{\prime}}(\Omega)} \\
& \quad \leq\|f \psi\|_{L^{m}(\Omega)}\left\|k^{-h}\right\|_{L^{1}(\Omega)}^{\frac{1}{(h+1) m^{\prime}}}\left\|k\left|u_{n}\right|^{\frac{[p(\gamma-1)+1](h+1) m^{\prime}}{h}} \psi^{\frac{(h+1) m^{\prime}(\eta-1)}{h}}\right\|_{L^{1}(\Omega)}^{\frac{h}{(h+1) m^{\prime}}}
\end{aligned}
$$

Then, we choose $\gamma$ and $\eta$ such that

$$
\begin{gathered}
\lambda+p(\gamma-1)+1 \geq \max \left\{\frac{p(h+1) r \gamma}{(r-p) h}, \frac{[p(\gamma-1)+1](h+1) m^{\prime}}{h}\right\}, \\
\eta \leq \min \left\{\frac{(h+1) r(\eta-p)}{(r-p) h}, \frac{(h+1) m^{\prime}(\eta-1)}{h}\right\} .
\end{gathered}
$$


For this purpose, we have to impose that $\lambda>\underline{\lambda}, \frac{1}{p^{\prime}}<\gamma \leq \tilde{\gamma}$ and $\eta \geq \tilde{\eta}_{0}$, where

$$
\begin{gathered}
\tilde{\gamma}=\min \left\{\frac{(\lambda-p+1) h(r-p)}{p(p h+r)}, \frac{\lambda h(m-1)+(p-1)(h+m)}{p(h+m)}\right\}, \\
\tilde{\eta}_{0}=\max \left\{\frac{p(h+1) r}{p h+r}, \frac{(h+1) m}{h+m}\right\} .
\end{gathered}
$$

Thus, we apply Young inequality in (3.9)-(3.11). Putting together the estimates obtained in this way with (3.8) and using (1.13) and the fact that $0 \leq \psi \leq 1$ in $\Omega$, we deduce that

$$
\left\|K\left(\cdot, u_{n}\right)\left|u_{n}\right|^{p(\gamma-1)+1} \psi^{\tilde{\eta}_{0}}\right\|_{L^{1}(\Omega)} \leq C_{3} \quad \forall \frac{1}{p^{\prime}}<\gamma \leq \tilde{\gamma},
$$

where $C_{3}$ is a positive constant not depending on $n$. Since $\tilde{\lambda}=\lambda+p(\tilde{\gamma}-1)+1$, in particular, we deduce that

$$
\left\|K\left(\cdot, u_{n}\right)\left|u_{n}\right|^{\tilde{\lambda}-\lambda} \psi^{\tilde{\eta}_{0}}\right\|_{L^{1}(\Omega)} \leq C_{3} .
$$

Moreover, going back to estimate (3.7), we also deduce that the quantity

$$
\int_{\Omega}\left|D w_{n}\right|^{p}\left(\delta+\left|w_{n}\right|\right)^{p(\gamma-1)}
$$

is uniformly bounded with respect to $n$.

Now, we observe that, if $\lambda \geq \bar{\lambda}$, then $\tilde{\gamma} \geq 1$ and, choosing $\gamma=1$, we get the result with $\tilde{p}=p$. Otherwise, if $\underline{\lambda}<\lambda<\overline{\bar{\lambda}}$, then $\frac{1}{p^{\prime}}<\tilde{\gamma}<1$. In this case, for any fixed $1 \leq q<p$, using Hölder inequality, we obtain that

$$
\begin{aligned}
& \int_{\Omega}\left|D u_{n}\right|^{q} \psi^{\tilde{\eta}_{0}}=\int_{\Omega} \frac{\left|D w_{n}\right|^{q}}{\left(\delta+\left|w_{n}\right|\right)^{q(1-\tilde{\gamma})}}\left(\delta+\left|w_{n}\right|\right)^{q(1-\tilde{\gamma})} \psi^{\tilde{\eta}_{0}} \\
& \leq\left[\int_{\Omega} \frac{\left|D w_{n}\right|^{p}}{\left(\delta+\left|w_{n}\right|\right)^{p(1-\tilde{\gamma})}}\right]^{\frac{q}{p}}\left\|\left|u_{n}\right|^{q(1-\tilde{\gamma})} \psi^{\tilde{\eta}_{0}}\right\|_{L^{\frac{p}{p-q}}(\Omega)} \\
& \leq\left[\int_{\Omega} \frac{\left|D w_{n}\right|^{p}}{\left(\delta+\left|w_{n}\right|\right)^{p(1-\tilde{\gamma})}}\right]^{\frac{q}{p}}\left\|k^{-h}\right\|_{L^{1}(\Omega)}^{\frac{p-q}{p(h+1)}}\left\|\left.|k| u_{n}\right|^{\frac{p q(h+1)(1-\tilde{\gamma})}{(p-q) h}} \psi^{\tilde{\eta}_{0}}\right\|_{L^{1}(\Omega)}^{\frac{(p-q) h}{p(h+1)}} .
\end{aligned}
$$

Thus, the right-hand side of the previous inequality is uniformly bounded with respect to $n$ if $\frac{p q(h+1)(1-\tilde{\gamma})}{(p-q) h}=\tilde{\lambda}$, that is,

$$
q=\frac{p \tilde{\lambda} h}{(\lambda+1)(h+1)-\tilde{\lambda}}=\min \left\{\frac{(\lambda-p+1) h r}{(\lambda+1) h+r}, \frac{p \lambda h m}{(\lambda+1) h+m}\right\} .
$$

Lemma 3.4. Assume (1.2), (1.3) with $b \in L^{N, 1}(\Omega)$, (1.8), (1.9), (1.12), (1.13) and (3.4). Assume also that $\lambda>\underline{\lambda}$, where $\underline{\lambda}$ is as in (1.17) but with $r=N$. Then, there exists $\tilde{\eta}_{1}>1$ depending only on $\psi, h, N$ and $p$, such that the sequences $\left\{\left|D u_{n}\right|^{\tilde{q}_{1}} \psi^{\tilde{\eta}_{1}}\right\}$ and $\left\{K\left(\cdot, u_{n}\right) \psi^{\tilde{\eta}_{1}}\right\}$ are bounded in $L^{1}(\Omega)$, where $\tilde{q}_{1}$ is defined in (1.21). Moreover, if (3.3) is fulfilled, then there exists $\tilde{\eta}_{2}>1$ depending only on $\psi, h, m, N$ and $p$, such that the sequences $\left\{\left|D u_{n}\right| \tilde{p} \psi^{\tilde{\eta}_{2}}\right\}$ 
and $\left\{K\left(\cdot, u_{n}\right)\left|u_{n}\right|^{\tilde{\lambda}-\lambda} \psi^{\tilde{\eta}_{2}}\right\}$ are bounded in $L^{1}(\Omega)$, where $\bar{\lambda}, \tilde{\lambda}, \tilde{q}$ and $\tilde{p}$ are as in (1.18)-(1.20) and (3.5) but with $r=N$.

Proof. The proof is divided into two steps.

STEP I. We fix $0<\theta<\frac{1}{p^{\prime}}, \eta>p+\frac{(r-p) h}{(h+1) r}$ and we choose

$$
v \psi^{\eta}=\left[1-\frac{1}{\left(1+\left|u_{n}\right|\right)^{p(1-\theta)-1}}\right] \operatorname{sign}\left(u_{n}\right) \psi^{\eta}
$$

as a test function in $(3.2)$. Notice that $p(1-\theta)-1>0$ if and only if $\theta<\frac{1}{p^{\prime}}$. Since

$$
D\left(v \psi^{\eta}\right)=[p(1-\theta)-1] \frac{D u_{n}}{\left(1+\left|u_{n}\right|\right)^{p(1-\theta)}} \psi^{\eta}+\eta D \psi v \psi^{\eta-1},
$$

exploiting (1.2), (2.6), (1.3) and the fact that $|v| \leq 1,|v| \psi^{\eta} \leq 1$ a.e. in $\Omega$, we obtain that

$$
\begin{aligned}
& \alpha[p(1-\theta)-1] \int_{\Omega} \frac{\left|D u_{n}\right|^{p}}{\left(1+\left|u_{n}\right|\right)^{p(1-\theta)}} \psi^{\eta}+\int_{\Omega} K\left(x, u_{n}\right) v \psi^{\eta} \\
& \leq \beta \eta\||D \psi|\|_{L^{\infty}(\Omega)} \int_{\Omega}\left|D u_{n}\right|^{p-1} \psi^{\eta-1}+\int_{\Omega}|b(x)|\left|D u_{n}\right|^{p-1} \psi^{\eta}+\|f\|_{L^{1}(\Omega)},
\end{aligned}
$$

which, using (1.13), implies that

$$
\begin{gathered}
C_{0} \int_{\Omega}\left|D\left[\left(1+\left|u_{n}\right|\right)^{\theta}-1\right]\right|^{p} \psi^{\eta}+|| k\left|u_{n}\right|^{\lambda} \psi^{\eta} \|_{L^{1}(\Omega)} \\
\leq C_{1} \int_{\Omega}\left|D\left[\left(1+\left|u_{n}\right|\right)^{\theta}-1\right]\right|^{p-1}\left(1+\left|u_{n}\right|\right)^{(p-1)(1-\theta)} \psi^{\eta-1} \\
+C_{2} \int_{\Omega}|b(x)|\left|D\left[\left(1+\left|u_{n}\right|\right)^{\theta}-1\right] \operatorname{sign}\left(u_{n}\right)\right|^{p-1}\left(1+\left|u_{n}\right|\right)^{(p-1)(1-\theta)} \psi^{\eta} \\
+|| k\left|u_{n}\right|^{\lambda-p(1-\theta)+1} \psi^{\eta}\left\|_{L^{1}(\Omega)}+\right\| f \|_{L^{1}(\Omega)},
\end{gathered}
$$

where $C_{0}=\frac{\alpha[p(1-\theta)-1]}{\theta^{p}}, C_{1}=\frac{\beta \eta\||D \psi|\|_{L^{\infty}(\Omega)}}{\theta^{p-1}}$ and $C_{2}=\frac{1}{\theta^{p-1}}$. Thanks to Young inequality, the right-hand side of (3.16) can be estimated by

$$
\begin{aligned}
& \frac{C_{0}}{p^{\prime}} \int_{\Omega}\left|D\left[\left(1+\left|u_{n}\right|\right)^{\theta}-1\right]\right|^{p} \psi^{\eta} \\
+ & \frac{2^{p-1} C_{1}^{p}}{p C_{0}^{p-1}} \int_{\Omega}\left(1+\left|u_{n}\right|\right)^{p(p-1)(1-\theta)} \psi^{\eta-p}+\frac{2^{p-1} C_{2}^{p}}{p C_{0}^{p-1}} \int_{\Omega}|b(x)|^{p}\left(1+\left|u_{n}\right|\right)^{p(p-1)(1-\theta)} \psi^{\eta} \\
& +\frac{1}{2}\left\|k\left|u_{n}\right|^{\lambda} \psi^{\eta}\right\|_{L^{1}(\Omega)}+c_{3},
\end{aligned}
$$


where $C_{3}$ is a positive constant that does not depend on $n$. Hence, from (3.16) we get

$$
\begin{aligned}
& C_{4} \int_{\Omega}\left|D\left[\left(1+\left|u_{n}\right|\right)^{\theta}-1\right]\right|^{p} \psi^{\eta}+\frac{1}{2}\left\|k\left|u_{n}\right|^{\lambda} \psi^{\eta}\right\|_{L^{1}(\Omega)} \\
& \leq C_{5} \int_{\Omega}\left(1+\left|u_{n}\right|\right)^{p(p-1)(1-\theta)} \psi^{\eta-p} \\
&+C_{6} \int_{\Omega}|b(x)|^{p}\left(1+\left|u_{n}\right|\right)^{p(p-1)(1-\theta)} \psi^{\eta}+C_{3}
\end{aligned}
$$

where $C_{4}=\frac{C_{0}}{p}, C_{5}=\frac{2^{p-1} C_{1}^{p}}{p C_{0}^{p-1}}$ and $C_{6}=\frac{2^{p-1} C_{2}^{p}}{p C_{0}^{p-1}}$. Using Hölder inequality and (1.14), we have that

$$
\begin{gathered}
C_{5} \int_{\Omega}\left(1+\left|u_{n}\right|\right)^{p(p-1)(1-\theta)} \psi^{\eta-p} \leq C_{5}|\Omega|^{\frac{p}{N}}\left\|\left(1+\left|u_{n}\right|\right)^{p(p-1)(1-\theta)} \psi^{\eta-p}\right\|_{L^{\frac{N}{N-p}}(\Omega)} \\
\leq C_{5}|\Omega|^{\frac{p}{N}}\left\|\left(k^{-1} \psi\right)^{h}\right\|_{L^{1}(\Omega)}^{\frac{N-p}{(h+1) N}} \\
\times\left\|k\left(1+\left|u_{n}\right|\right)^{\frac{p(p-1)(h+1) N(1-\theta)}{h(N-p)}} \psi^{\frac{(h+1) N(\eta-p)}{h(N-p)}-1}\right\|_{L^{1}(\Omega)}^{\frac{h(N-p)}{(h+1) N}}, \quad(3.18)
\end{gathered}
$$

and

$$
\begin{gathered}
C_{6} \int_{\Omega}|b(x)|^{p}\left(1+\left|u_{n}\right|\right)^{p(p-1)(1-\theta)} \psi^{\eta} \leq C_{6}\|b\|_{L^{N}(\Omega)}^{p}\left\|\left(1+\left|u_{n}\right|\right)^{p \gamma} \psi^{\eta}\right\|_{L^{\frac{N}{N-p}}(\Omega)} \\
\leq C_{6}\|b\|_{L^{N}(\Omega)}^{p}\left\|\left(k^{-1} \psi\right)^{h}\right\|_{L^{1}(\Omega)}^{\frac{N-p}{(h+1) N}} \\
\times\left\|k\left(1+\left|u_{n}\right|\right)^{\frac{p(p-1)(h+1) N(1-\theta)}{h(N-p)}} \psi^{\frac{(h+1) N \eta}{h(N-p)}-1}\right\|_{L^{1}(\Omega)}^{\frac{h(N-p)}{(h+1) N}} \cdot
\end{gathered}
$$

Then, we choose $\theta$ and $\eta$ such that

$$
\begin{gathered}
\frac{p(p-1)(h+1) N(1-\theta)}{h(N-p)} \leq \lambda, \\
\frac{(h+1) N(\eta-p)}{h(N-p)}-1 \geq \eta .
\end{gathered}
$$

For this purpose, we have to impose that $\lambda>\underline{\lambda}, \tilde{\theta} \leq \theta<\frac{1}{p^{\prime}}$ and $\eta \geq \tilde{\eta}_{1}$, where $\underline{\lambda}$ is as in (1.17) but with $r=N$ and

$$
\begin{gathered}
\tilde{\theta}=1-\frac{(N-p) \lambda h}{p(p-1)(h+1) N}, \\
\tilde{\eta}_{1}=\frac{[(p+1) N-p] h+p N}{p h+N} .
\end{gathered}
$$

Thus, we apply Young inequality in (3.18) and (3.19). Putting together the estimates obtained in this way with (3.17) and using the fact that $0 \leq \psi \leq 1$ 
in $\Omega$, we deduce that

$$
\begin{aligned}
\int_{\Omega}\left|D\left[\left(1+\left|u_{n}\right|\right)^{\theta}-1\right]\right|^{p} \psi^{\tilde{\eta}_{1}}+|| k\left|u_{n}\right|^{\lambda} \psi^{\tilde{\eta}_{1}} \|_{L^{1}(\Omega)} & \\
& \leq C_{7} \quad \forall \tilde{\theta} \leq \theta<\frac{1}{p^{\prime}} .
\end{aligned}
$$

where $C_{7}$ is a positive constant which not depending on $n$.

Now, for any fixed $1 \leq q<p$, using Hölder inequality, (1.14) and (3.20), we obtain that

$$
\begin{aligned}
\int_{\Omega}\left|D u_{n}\right|^{q} \psi^{\tilde{\eta}_{1}} & =\frac{1}{\theta^{q}} \int_{\Omega}\left|D\left[\left(1+\left|u_{n}\right|\right)^{\theta}-1\right]\right|^{q}\left(1+\left|u_{n}\right|\right)^{q(1-\theta)} \psi^{\tilde{\eta}_{1}} \\
& \leq \frac{C_{7}^{\frac{q}{p}}}{\theta^{q}}\left\|\left(1+\left|u_{n}\right|\right)^{q(1-\theta)} \psi^{\tilde{\eta}_{1}}\right\|_{L^{\frac{p}{p-q}}(\Omega)} \\
\leq & \frac{C_{7}^{\frac{q}{p}}}{\theta^{q}}\left\|\left(k^{-1} \psi\right)^{h}\right\|_{L^{1}(\Omega)}^{\frac{p-q}{p(1)}}\left\|k\left(1+\left|u_{n}\right|\right)^{\frac{p q(h+1)(1-\theta)}{(p-q) h}} \psi^{\tilde{\eta}_{1}}\right\|_{L^{1}(\Omega)}^{\frac{(p-q) h}{p(h+1)}}
\end{aligned} .
$$

Thanks to (3.20), the right-hand side of the previous inequality is uniformly bounded with respect to $n$ if $\frac{p q(h+1)(1-\theta)}{(p-q) h} \leq \lambda$, that is,

$$
q \leq \frac{p \lambda h}{[\lambda+p(1-\theta)] h+p(1-\theta)} .
$$

Hence, for any $1 \leq q<\tilde{q}_{1}$ where $\tilde{q}_{1}$ is defined in (1.21), we can choose $\tilde{\theta} \leq \theta<\frac{1}{p^{\prime}}$ sufficiently close to $\frac{1}{p^{\prime}}$ in such a way that (3.21) is fulfilled.

STEP II. Assume (2.10). Arguing as in the proof of Lemma 3.3, we deduce that for every $\gamma>\frac{1}{p^{\prime}}$

$$
\begin{aligned}
C_{8} \int_{\Omega}\left|D u_{n}\right|^{p}\left(\delta+\left|u_{n}\right|\right)^{p(\gamma-1)} \psi^{\eta} & +\int_{\Omega}\left|K\left(x, u_{n}\right)\right|\left|u_{n}\right|^{p(\gamma-1)+1} \psi^{\eta} \\
\leq C_{9} \int_{\Omega}\left(\delta+\left|u_{n}\right|\right)^{p \gamma} \psi^{\eta-p} & +C_{10} \int_{\Omega}|b(x)|^{p}\left(\delta+\left|u_{n}\right|\right)^{p \gamma} \psi^{\eta} \\
& +\int_{\Omega}|f(x)|\left(\delta+\left|u_{n}\right|\right)^{p(\gamma-1)+1} \psi^{\eta}
\end{aligned}
$$

Using Hölder inequality, we have that

$$
\begin{aligned}
& C_{9} \int_{\Omega}\left(\delta+\left|u_{n}\right|\right)^{p \gamma} \psi^{\eta-p} \leq C_{9}|\Omega|^{\frac{p}{N}}\left\|\left(\delta+\left|u_{n}\right|\right)^{p \gamma} \psi^{\eta-p}\right\|_{L^{\frac{N}{N-p}}(\Omega)} \\
& \leq C_{9}|\Omega|^{\frac{p}{N}}\left\|\left(k^{-1} \psi\right)^{h}\right\|_{L^{1}(\Omega)}^{\frac{N-p}{(h+1) N}}\left\|k\left|u_{n}\right|^{\frac{p(h+1) N \gamma}{(N-p) h}} \psi^{\frac{(h+1) N(\eta-p)}{(N-p) h}}-1\right\|_{L^{1}(\Omega)}^{\frac{(N-p) h}{(h+1) N}}, \\
& C_{10} \int_{\Omega}|b(x)|^{p}\left(\delta+\left|u_{n}\right|\right)^{p \gamma} \psi^{\eta} \leq C_{10}\|b\|_{L^{N}(\Omega)}^{p}\left\|\left(\delta+\left|u_{n}\right|\right)^{p \gamma} \psi^{\eta}\right\|_{L^{\frac{N}{N-p}}}(\Omega) \\
& \leq C_{10}\|b\|_{L^{N}(\Omega)}^{p}\left\|\left(k^{-1} \psi\right)^{h}\right\|_{L^{1}(\Omega)}^{\frac{N-p}{(h+1) N}}\left\|k\left|u_{n}\right|^{\frac{p(h+1) N \gamma}{h(N-p)}} \psi^{\frac{(h+1) N \eta}{(N-p) h}-1}\right\|_{L^{1}(\Omega)}^{\frac{(N-p) h}{(h+1) N}}
\end{aligned}
$$


and

$$
\begin{aligned}
\left.\int_{\Omega}\left|f(x)\left\|\left.u_{n}\right|^{p(\gamma-1)+1} \psi^{\eta} \leq\right\| f \psi\left\|_{L^{m}(\Omega)}\right\|\right| u_{n}\right|^{p(\gamma-1)+1} \psi^{\eta-1} \|_{L^{m^{\prime}}(\Omega)} \\
\leq\|f \psi\|_{L^{m}(\Omega)}\left\|\left(k^{-1} \psi\right)^{h}\right\|_{L^{1}(\Omega)}^{\frac{1}{(h+1) m^{\prime}}} \\
\times\left\|k\left|u_{n}\right|^{\frac{[p(\gamma-1)+1](h+1) m^{\prime}}{h}} \psi^{\frac{(h+1) m^{\prime}(\eta-1)}{h}-1}\right\|_{L^{1}(\Omega)}^{\frac{h}{(h+1) m^{\prime}}}
\end{aligned}
$$

Then, we choose $\gamma$ and $\eta$ such that

$$
\begin{gathered}
\lambda+p(\gamma-1)+1 \geq \max \left\{\frac{p(h+1) N \gamma}{(N-p) h}, \frac{[p(\gamma-1)+1](h+1) m^{\prime}}{h}\right\}, \\
\eta \leq \min \left\{\frac{(h+1) N(\eta-p)}{h(N-p)}-1, \frac{(h+1) m^{\prime}(\eta-1)}{h}-1\right\}
\end{gathered}
$$

Hence, we impose that $\lambda>\underline{\lambda}, \frac{1}{p^{\prime}}<\gamma \leq \tilde{\gamma}$ and $\eta \geq \tilde{\eta}_{2}$, where $\tilde{\gamma}$ is as in (3.12) but with with $r=N$, and

$$
\tilde{\eta}_{2}=\max \left\{\frac{[(p+1) N-p] h+p N}{p h+N}, \frac{(h+1) m}{h+m}\right\} .
$$

The result now follows proceeding as in the proof of Lemma 3.3.

\subsection{Proof of Theorems 3.1 and 3.2}

Proof of Theorem 3.1. Let $\left\{u_{n}\right\}$ be the sequence of weak solutions of approximate problems (3.1) constructed above. The result is an immediate consequence of Lemma 3.3, since, arguing as in the proof of Theorem 2.3 in [10], we can prove that there exists a weak solution $u$ of (1.11) such that, up to a subsequence, $u_{n} \rightarrow u$ and $D u_{n} \rightarrow D u$ a.e. in $\Omega$.

Proof of Theorem 3.2. Let $\left\{u_{n}\right\}$ be the sequence of weak solutions of approximate problems (3.1) constructed above. Closely following the outline of the proof of Theorem 2.3 in [4], we can prove that

$$
\begin{cases}\left\{u_{n}\right\} & \text { is bounded in } M^{\frac{p^{*}}{p^{\prime}}}(\Omega), \\ \left\{\left|D u_{n}\right|\right\} & \text { is bounded in } M^{N^{\prime}(p-1)}(\Omega) .\end{cases}
$$

In order to perform the limit process and deduce the existence of a weak solution $u$ of (1.11) that satisfies (1.5), we just have to prove that the sequence $\left\{K\left(\cdot, u_{n}\right)\right\}$ is uniformly integrable on $\Omega$, since the other terms can be treated as in the proof of Theorem 2.3 in [4].

We fix $\sigma \geq 0, \tau>0$ and we choose

$$
v_{\tau}=\frac{T_{\tau}\left(G_{\sigma}\left(u_{n}\right)\right)}{\tau}
$$

as a test function in (2.7), where $T_{\tau}$ and $G_{\sigma}$ are defined in (2.5) and (2.33), respectively. Notice that

$$
\left|v_{\tau}\right| \leq \chi_{A_{n, \sigma}} \quad \text { a.e. in } \Omega,
$$


where

$$
A_{n, \sigma}=\left\{x \in \Omega:\left|u_{n}(x)\right|>\sigma\right\},
$$

and, for any subset $U \subset \mathbb{R}^{N}, \chi_{U}$ denotes the characteristic function of $U$. By (1.2), (2.6) and (1.3), we obtain that

$$
\begin{aligned}
\frac{\alpha}{\tau}\left\|\left|D T_{\tau}\left[G_{\sigma}\left(u_{n}\right)\right]\right|\right\|_{L^{p}(\Omega)}^{p}+\| K(\cdot, & \left.u_{n}\right) v_{\tau} \|_{L^{1}(\Omega)} \\
& \leq \int_{\Omega}|b(x)|\left|D u_{n}\right|^{p-1}\left|v_{\tau}\right|+\int_{\Omega}\left|f(x) \| v_{\tau}\right| .
\end{aligned}
$$

Dropping the positive term coming from the principal part and using (3.27), we get

$$
\left\|K\left(\cdot, u_{n}\right) v_{\tau}\right\|_{L^{1}\left(A_{n, \sigma}\right)} \leq \int_{A_{n, \sigma}}|b(x)|\left|D u_{n}\right|^{p-1}+\|f\|_{L^{1}\left(A_{n, \sigma}\right)} .
$$

By the generalized Hölder inequality, we have that

$\int_{A_{n, h}}|b(x)|\left|D u_{n}\right|^{p-1} \leq\|b\|_{L^{N, 1}\left(A_{n, \sigma}\right)}\left\|\left|D u_{n}\right|^{p-1}\right\|_{L^{N^{\prime}, \infty}(\Omega)} \leq C_{0}\|b\|_{L^{N, 1}\left(A_{n, \sigma}\right)}$,

where $C_{0}$ is a positive constant that does not depend on $n$. Hence, from (3.29) we deduce that

$$
\left\|K\left(\cdot, u_{n}\right) v_{\tau}\right\|_{L^{1}\left(A_{n, \sigma}\right)} \leq C_{0}\|b\|_{L^{N, 1}\left(A_{n, \sigma}\right)}+\|f\|_{L^{1}\left(A_{n, \sigma}\right)} .
$$

We observe that $v_{\tau} \rightarrow \chi_{A_{n, \sigma}}$ a.e. in $\Omega$, as $\tau \rightarrow 0$. Therefore, letting $\tau \rightarrow 0$ and using Fatou Lemma, estimate (3.30) yields

$$
\left\|K\left(\cdot, u_{n}\right)\right\|_{L^{1}\left(A_{n, \sigma}\right)} \leq C_{0}\|b\|_{L^{N, 1}\left(A_{n, \sigma}\right)}+\|f\|_{L^{1}\left(A_{n, \sigma}\right)} .
$$

On the other hand, for any fixed measurable subset $U \subset \Omega$ and $\sigma>0$, using assumption (1.12), we have that

$$
\begin{aligned}
\left\|K\left(\cdot, u_{n}\right)\right\|_{L^{1}(U)} \leq \| K\left(\cdot, u_{n}\right) & \left\|_{L^{1}\left(U \cap\left\{\left|u_{n}\right| \leq \sigma\right\}\right)}+\right\| K\left(\cdot, u_{n}\right) \|_{L^{1}\left(A_{n, \sigma}\right)} \\
& \leq\left\|\sup _{|\tau| \leq \sigma}|K(\cdot, \tau)|\right\|_{L^{1}(U)}+\left\|K\left(\cdot, u_{n}\right)\right\|_{L^{1}\left(A_{n, \sigma}\right)} .
\end{aligned}
$$

which, together with (3.31), implies that

$$
\left\|K\left(\cdot, u_{n}\right)\right\|_{L^{1}(U)} \leq\left\|\sup _{|\tau| \leq \sigma} \mid K(\cdot, \tau)\right\|\left\|_{L^{1}(U)}+C_{0}\right\| b\left\|_{L^{N, 1}\left(A_{n, \sigma}\right)}+\right\| f \|_{L^{1}\left(A_{n, \sigma}\right)} .
$$

Since $\left|A_{n, \sigma}\right| \rightarrow 0$ uniformly with respect to $n$, as $\sigma \rightarrow \infty$, for every $\epsilon>0$ we can choose $\sigma$ sufficiently large in such a way that

$$
C_{0}\|b\|_{L^{N, 1}\left(A_{n, \sigma}\right)}+\|f\|_{L^{1}\left(A_{n, \sigma}\right)} \leq \epsilon \quad \forall n \in \mathbb{N} .
$$

Therefore, from (3.32) it follows that

$$
\lim _{|U| \rightarrow 0}\left\|K\left(\cdot, u_{n}\right)\right\|_{L^{1}(U)}=0 \quad \text { uniformly with respect to } n \text {. }
$$

Now, we can apply Lemma 3.4 and we get the result. 


\section{References}

[1] R.A. Adams. Sobolev spaces. Academic Press, New York, 1975.

[2] M.F. Betta, V. Ferone, A. Mercaldo. Regularity for solutions of nonlinear elliptic equations. Bull. Sci. Math., 118 (1994), 539-567.

[3] M.F. Betta, A. Mercaldo, F. Murat, M.M. Porzio. Uniqueness of renormalized solutions to nonlinear elliptic equations with lower order term and right-hand side in $L^{1}(\Omega)$. ESAIM Control Optim. Calc. Var., 8 (2002), 239-272 (special issue dedicated to the memory of Jacques-Louis Lions).

[4] M.F. Betta, A. Mercaldo, F. Murat, M.M. Porzio. Existence of renormalized solutions to nonlinear elliptic equations with a lower-order term and righthand side a measure. J. Math. Pures Appl., 82 (2003), 90-124.

[5] M.F. Betta, A. Mercaldo, F. Murat, M.M. Porzio. Uniqueness results for nonlinear elliptic equations with a lower order term. Nonlinear Anal. 63 (2005) 153-170.

[6] L. Boccardo, T. Gallouët. Nonlinear elliptic equations with rigth hand side measures. Comm. Partial Diff. Equations, 17 (1992), 641-655.

[7] L. Boccardo, D. Giachetti. Some remarks on the regularity of solutions of strongly nonlinear problems and applications. Ricerche Mat. 34 (1985), 309323.

[8] L. Boccardo, T. Leonori. Local properties of solutions of elliptic equations depending on local properties of the data. Methods Appl. Anal., 15 (2008), 53-63.

[9] G. Bottaro, M. E. Marina. Problema di Dirichlet per equazioni ellittiche di tipo variazionale su insiemi non limitati. Boll. Un. Mat. Ital., 8 (1973), 46-56.

[10] F. Clemente. Existence and regularity results to nonlinear elliptic equations with lower order terms. (under review)

[11] G. Dal Maso, F. Murat, L. Orsina, A. Prignet. Renormalized solutions for elliptic equations with general measure data. Ann. Scuola Norm. Sup. Pisa Cl. Sci., 28 (1999), 741-808.

[12] T. Del Vecchio. Nonlinear elliptic equations with measure data. Potential Analysis, 4 (1995), 185-203.

[13] T. Del Vecchio, M.M. Porzio. Existence results for a class of non coercive Dirichlet problems. Ric. Mat., 44 (1995), 421-438.

[14] T. Del Vecchio, M.R. Posteraro. Existence and regularity results for nonlinear elliptic equations with measure data. Adv. Diff. Eq., 1 (1996), 899-917.

[15] J. Leray, J.-L. Lions. Quelques résulatats de Višik sur les problèmes elliptiques nonlinéaires par les méthodes de Minty-Browder. Bull. Soc. Math. France, 93 (1965), 97-107.

[16] F. Murat. Soluciones renormalizadas de EDP ellipticas no lineales. Preprint 93023, Laboratoire d'Analyse Numérique de l'Université Paris VI, 1993.

[17] F. Murat. Equations elliptiques non linéaires avec second membre $L^{1}$ ou measure, in: Actes du 26éme Congrés National d'Analyse Numérique, Les Karellis, France, 1994, pp. A12-A24.

[18] J. Serrin. Pathological solutions of elliptic differential equations. Ann. Sc. Norm. Sup. Pisa, 18 (1964), 385-387. 
[19] G. Stampacchia. Régularization des solutions de problèmes aux limites elliptiques à données discontinues. Inter. Symp. on Lin. Spaces, Jerusalem (1960), 399-408.

[20] G. Stampacchia. Some limit cases of $L^{p}$-estimates for solutions of second order elliptic equations. Comm. Pre Appl. Math., 16 (1963), 505-510.

[21] G. Stampacchia. Le probléme de Dirchlet pour les équations elliptiques du second ordre à coefficients discontinus. Ann. Inst. Fourier (Grenoble), 15 (1965), 189-258.

Francesco Clemente

Dipartimento di Matematica "Guido Castelnuovo"

Sapienza Università di Roma

P.le A. Moro 2

00185 Rome

Italy

e-mail: clemente@mat.uniroma1.it 